Supplementary Information (SI) for

\title{
Significant but spatiotemporal-heterogeneous health risks caused by airborne exposure to multiple toxic trace elements in China
}

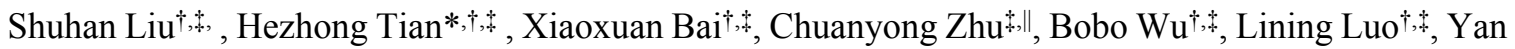
$\mathrm{Hao}^{\dagger}$, Wei Liu ${ }^{\dagger, \ddagger}$, Shumin Lin ${ }^{\dagger, \ddagger}$, Shuang Zhao ${ }^{\dagger, \star}$, Kun Wang ${ }^{\dagger, \nabla}$, Kaiyun Liu ${ }^{\dagger, \S}$, Jiajia Gao ${ }^{\dagger, \nabla}$, Qiang Zhang* ${ }^{*}$, Kai Zhang ${ }^{\omega}$, Haidong $\mathrm{Kan}^{\Psi}$, Yang Liu ${ }^{\Omega}$, Jiming Hao*,§

$\uparrow$ State Key Joint Laboratory of Environmental Simulation \& Pollution Control, School of Environment, Beijing Normal University, Beijing 100875, China.

$\$$ Center for Atmospheric Environmental Studies, Beijing Normal University, Beijing 100875 , China.

$\S$ School of Environment, Tsinghua University, Beijing 100084, China.

${ }^{\perp}$ Department of Earth System Science, Tsinghua University, 100084 Beijing, China.

\| College of Environmental Science and Engineering, Qilu University of Technology (Shandong Academy of Sciences), Jinan, 250353, China.

${ }^{\omega}$ Department of Environmental Health Sciences School of Public Health University at Albany, State University of New York One University Place Rensselaer, NY 12144.

${ }^{\Psi}$ School of Public Health, Fudan University, Shanghai 200032, China.

${ }^{\Omega}$ Rollins School of Public Health, Emory University, Atlanta 30322, GA, USA.

${ }^{\nabla}$ Department of Air Pollution Control, Beijing Municipal Institute of Labour Protection, Beijing 100054, China.

*Hezhong Tian, Jiming Hao, Qiang Zhang

Email: H.T. (email: hztian@bnu.edu.cn); J.H. (email: hjm-den@tsinghua.edu.cn); Q.Z. (email: qiangzhang@tsinghua.edu.cn). 
This Supporting Information contains 51 pages, 14 tables and 26 figures.

Table of Contents

Tables:

Table S1. Summary of trace element species and the associated emission source categories S10

Table S2. Data sources of activity data for the trace element emission sectors in China. S11

Table S3. Spatial allocation method of TEs emission for each source. S12

Table S4. Comparison of meteorological predictions vs. observations over China, 2012 ..................S13

Table S5. Comparison of meteorological predictions vs. observations over China, 2015

Table S6 Model performance on $\mathrm{PM}_{2.5}$ and trace elements in January, April, August and October over

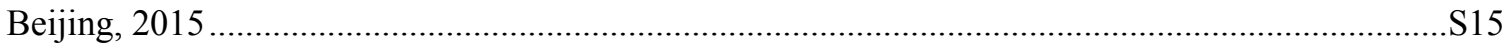

Table S7. Infiltration factors and annual mean amount of time indoors and outdoors ........................S16

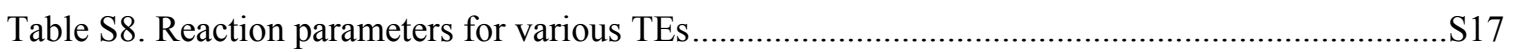

Table S9. Probability distribution for each parameter for uncertainty analysis..................................S18

Table S10. Uncertainties in the sectoral emissions of trace elements in China, 2015 .........................S19

Table S11. Emissions of trace elements in China 2015 by province $(\mathrm{t})$..............................................S20

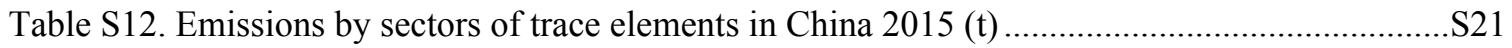

Table S13. Spatial correlations among 11TEs and as well as with population ..................................S22

Table S14. The population-weighted concentrations (PWC) and the maximum gridded concentrations (MGC) of 11 TEs in different regions of China in 2012 and 2015 ..................................................S23

\section{Figures:}

Figure S1. Time series of observed and predicted daily concentrations for $\mathrm{PM}_{2.5}\left(\mu \mathrm{g} / \mathrm{m}^{3}\right)$ and trace elements $\left(\mathrm{ng} / \mathrm{m}^{3}\right)$ in BNU site in January, 2015.

Figure S2. Time series of observed and predicted daily concentrations for $\mathrm{PM}_{2.5}\left(\mu \mathrm{g} / \mathrm{m}^{3}\right)$ and trace elements $\left(\mathrm{ng} / \mathrm{m}^{3}\right)$ in BNU site in April, 2015

Figure S3. Time series of observed and predicted daily concentrations for $\mathrm{PM}_{2.5}\left(\mu \mathrm{g} / \mathrm{m}^{3}\right)$ and trace elements ( $\left.\mathrm{ng} / \mathrm{m}^{3}\right)$ in BNU site in August, 2015.

Figure S4. Time series of observed and predicted daily concentrations for $\mathrm{PM}_{2.5}\left(\mu \mathrm{g} / \mathrm{m}^{3}\right)$ and trace elements $\left(\mathrm{ng} / \mathrm{m}^{3}\right)$ in BNU site in October, 2015

Figure S5. Comparison between simulated and observed TEs concentrations in multiple cities for 2012. S28

Figure S6. Comparison between simulated and observed TEs concentrations in multiple cities for 2015.

Figure S7. Comparison of spatial variations between simulated and surface observed $\mathrm{PM}_{2.5}$ concentrations for the year of 2015 in mainland China.

Figure S8. Uncertainty analysis of TEs emissions in China, 2015. 
Figure S9. Spatial distribution characteristics of TEs emissions in 2015.

Figure S10. Spatial distribution of activity level of important industries in China in 2015

Figure S11. Provincial TEs emissions from anthropogenic sources and national composition by source categories in 2015 S34

Figure $\mathrm{S} 12 . \mathrm{PM}_{2.5}$ emission changes in China and the six regions of China. S35

Figure S13. Comparisons of the total TEs emissions in China in the years of 2012 and 2015 by region S36

Figure S14. Comparisons of each trace element emissions by sectors in 2012 and 2015. S37

Figure S15. Emission reductions by sectors of each trace element from 2012-2015 in China. Purple

dots on lines in each figure represent total reduction of each trace element S38

Figure S16. Spatial variations of the reductions of TEs emissions in China from 2012 to 2015 by trace element (2012 emissions minus 2015 emissions)

Figure S17. Spatiotemporal variations of Cd, Co, Sb, As, Se and Ni in China, 2012. Spatial

distribution of averaged seasonal concentrations of $\mathrm{Cd}, \mathrm{Co}, \mathrm{Sb}, \mathrm{As}, \mathrm{Se}$ and $\mathrm{Ni}$ in China, 2012 .........S40 Figure S18. Spatiotemporal variations of $\mathrm{Cr}, \mathrm{Cu}, \mathrm{Mn}, \mathrm{Pb}$ and $\mathrm{Zn}$ in China, 2012. Spatial distribution of averaged seasonal concentrations of $\mathrm{Cr}, \mathrm{Cu}, \mathrm{Mn}, \mathrm{Pb}$ and $\mathrm{Zn}$ in China, 2012.

Figure S19. Spatiotemporal variations of Cd, Co, Sb, As, Se and Ni in China, 2015. Spatial

distribution of averaged seasonal concentrations of Cd, Co, Sb, As, Se and Ni in China, 2015

Figure S20. Spatiotemporal variations of $\mathrm{Cr}, \mathrm{Cu}, \mathrm{Mn}, \mathrm{Pb}$ and $\mathrm{Zn}$ in China, 2015. Spatial distribution of averaged seasonal concentrations of $\mathrm{Cr}, \mathrm{Cu}, \mathrm{Mn}, \mathrm{Pb}$ and $\mathrm{Zn}$ in China, 2015.

Figure S21. Spatial variations of TEs concentrations in China from 2012 to 2015.

Figure S22. Annual surface observed $\mathrm{PM}_{2.5}$ concentrations of China, BTH, YRD, and PRD region from 2013 to 2015 . S45

Figure S23. Spatial distribution of ILCR value in 2012 for adults and children. S46

Figure S24. Spatial distribution of ILCR value in 2015 for adults and children S47

Figure S25. Spatial distribution of HI value in 2012 and 2015 . $\mathrm{S} 48$

Figure S26. Spatial distribution of people lived in the environment with HI exceeding 1 by TEs inhalation in 2012 (a) and 2015 (b). S49 
Supplementary Section 1

\section{Estimation methods and results of TEs Emission Inventory}

\section{Estimation methods}

The basic calculation of TEs emissions from coal combustion sources can be expressed by the following equation:

$E(t)=\sum_{i} \sum_{j} \sum_{k}\left[C_{i, j, k} \times A_{i, j, k} \times R_{i, j} \times P_{i j}\left(1-\eta P M_{(i, j)}\right)\left(1-\eta s o_{2(i, j)}\right)\left(1-\eta N O x_{(i, j)}\right)\right]$

where $E$ is the ambient emissions of cadmium, cobalt, antimony, arsenic, selenium, nickel, chromium, copper, manganese, lead, and zinc; $C$ is the averaged concentration of each trace element in feed coals in each province; $A$ is the amount of annual coal consumption; $R$ is the fraction of each trace element released with flue gas from varied coal combustion facilities; $\mathrm{P}$ is the proportion of a certain type of APCD; $\eta P M, \eta \mathrm{SO}_{2}$, and $\eta N O x$ represent the averaged fraction of one trace element which is removed from flue gas by the conventional $\mathrm{PM} / \mathrm{SO}_{2} / \mathrm{NOx}$ emission control devices, respectively; $i$ represents the province (autonomous region or municipality); $j$ represents the subcategory emission source; $k$ represents the type of coal as consumed (raw coal, cleaned coal, briquette, and coke); and $t$ represents the calendar year.

The averaged concentration values of cadmium, cobalt, antimony, arsenic, selenium, nickel, chromium, copper, manganese, lead, and zinc in feed coals on the provincial level, the release rates of these TEs from different combustion facilities, and the averaged removal efficiencies by different APCDs configurations reported in Tian et al. (2015) ${ }^{1}$ are adopted to calculate the final emissions in 2015 in this study. The amount of annual coal consumption is updated to 2015 according to the relevant statistical yearbooks, more detailed data sources are shown in Table S2.

The basic formulas of TEs emissions from non-coal combustion sources can be described as follows:

$$
E(t)=\sum_{i} \sum_{j}(A i, j \times E F j)
$$

Where $E$ is the ambient emissions of each trace element; $A$ is the annual production outputs of industrial producing processes, the amount of municipal solid wastes incineration, the ownership of vehicles, or liquid fuel consumption, etc.; $E F$ is the assumed average emission factors; $j$ is the specific emission source.

The averaged emission factors of cadmium, cobalt, antimony, arsenic, selenium, nickel, chromium, copper, manganese, lead, and zinc for the non-coal combustion sources reported in Tian et al. (2015) ${ }^{1}$ are adopted in this study. The detailed activity data for the non-coal sectors is updated to 2015 according to the relevant statistical yearbooks, as shown in Table S2. More detailed information about the calculation methods, data sources and gridded allocated methods of TEs emission inventory have been described in our previous studies. 


\section{TEs emissions in 2015}

As can be seen in Figure S9, the TEs emissions are mainly concentrated in northern, eastern, central and southern China, and this spatial pattern is highly consistent with the population distribution. Previous study indicated that $94 \%$ of population in China lives in southeast of the Hu Huanyong Line, where the area only accounts for approximately $43 \%$ of total land area in $\mathrm{China}^{2}$. The spatial pattern of densely populated southeast and sparsely populated northwest affects the distribution of important industries to some extent. Tian et al. (2015) indicated that the important industries such as coal-fired power plants, ferrous and nonferrous metal smelting sectors are mainly concentrated in eastern, central and southern, as well as the coastal cities of China. ${ }^{1}$

Figure S10 displays the spatial distribution of activity level of important industries in China in 2015. Coal consumption, outputs of industrial products in those densely populated and industrial regions is relatively higher than that in other regions, which may be mainly due to the increasing demands of human life and economic development. In addition, the activity level could directly determine the emissions of air pollutants. Therefore, the spatial distribution of 11 TEs emissions shares some similarity to distributions of the population, and is also related with the industrial structures and economic development.

Table S13 shows the spatial correlations among these 11TEs and as well as with population. As can be seen, basically all TEs are significantly correlated in spatial distribution. The correlation coefficients of $\mathrm{Sb}$ and $\mathrm{Cu}$ with other trace elements are relatively lower, which indicates that $\mathrm{Sb}$ and $\mathrm{Cu}$ may have some special sources different from other trace elements. The spatial distribution of all TEs are significantly correlated with population. It should be noted that, the correlation coefficients of $\mathrm{Sb}$ and $\mathrm{Cu}$ with population are relatively as high as 0.882 and 0.934 , respectively. As can be seen in Figure $\mathrm{S} 11, \mathrm{Cu}$ and $\mathrm{Sb}$ emissions mainly come from brake and tyre wear source, with notable higher emission intensity in urban areas than suburb and rural areas, which are allocated by regional population density (Table S3). Co, As, Se, Cr and Mn emissions are mainly from coal combustion sources for industrial production, which are mainly allocated by industrial gross domestic product (Table S3). So their correlation coefficients with population are relatively lower, which are less than 0.8 .

\section{Supplementary Section 2}

\section{Model evaluation}

As can be seen in Table S4 and S5, the simulated T2 in both 2012 and 2015 are slightly lower than observations except for November and December. The Mean bias (MB) values of T2 for June, and October to December are within the criteria $(\leq \pm 0.5 \mathrm{~K})$. The MB values of WS10 met the criteria $(\leq \pm 0.5 \mathrm{~m} / \mathrm{s})$ in January, June and July in both years, and root mean square error (RMSE) values are all basically a little higher than the criteria $(\leq 2.0)$. WS10 is over-predicted in all months in 2012 and 2015. 
MB values of WD10 are within the criteria $\left(\leq \pm 10^{\circ}\right)$ for January, September and October in both years. RH is generally over-predicted except for February, and October to December in both years. The NMB and NME values for these meteorological parameters are $-46.5 \% \sim 49.6 \%$, which are all within $\pm 50 \%$. This indicated that the WRF model simulation results could be acceptable and available. In addition, the WRF performance in this study is comparable to other studies using WRF in China. ${ }^{3-5}$ In general, the WRF simulations could well reproduce the real meteorological conditions in this study.

Due to the lack of official specific trace elements observations over China, daily observations of trace elements in January, April, August and October in 2012 and 2015 are obtained from the BNU monitoring site. Overall statistics of monthly chemical species concentrations over Beijing in 2015 is shown in Table S6. Model performs quite well in simulating $\mathrm{PM}_{2.5}$ concentrations. Correlation coefficients are relatively high, ranging from 0.7 in August to 0.9 in January. The NMBs are negative and between $-21 \%$ and $-45 \%$. The NMEs range from $25 \%$ to $46 \%$, which are all within the criteria of $\pm 50 \%$. TEs predictions have a relatively weaker ability than $\mathrm{PM}_{2.5}$ to match with observations, which may be partly attributed to their trace levels. Correlation coefficients range from 0.5 to 0.9 . The predictions for cobalt in January and October, antimony in April, arsenic in August, selenium in January and August, nickel in April and August, chromium in October, copper in April, zinc in April agree relatively well with the observations, with NMBs and NMEs all within $\pm 50 \%$. However, the NMBs or NMEs for the remaining are all slightly higher than the criteria, ranging from $-51 \%$ to $-78 \%$, and $51 \%$ to $91 \%$, respectively. Of all of the TEs simulations, cadmium simulations are the worst. Correlation coefficients are relatively lower, ranging from 0.4 to 0.6 . The NMBs are negative and between $-62 \%$ in August and $-181 \%$ in January. The NMEs range from $71 \%$ to $184 \%$.

Figure S1-S4 present the comparisons between daily chemical species concentration predictions and observations in January, April, August, and October in 2015, respectively. As for time dependency, $\mathrm{PM}_{2.5}$ predictions show the best agreement with daily observations in all months. The correlation coefficients range from 0.73 to 0.89 , which indicates that the model well reproduces the time trend and magnitude of $\mathrm{PM}_{2.5}$ concentrations. The correlation coefficients for TEs range from 0.54 to 0.87 in January, 0.46 to 0.85 in April, 0.44 to 0.84 in August, and 0.40 to 0.92 in October, respectively. Among all the TEs, antimony, arsenic, selenium, nickel, copper and lead predictions present the higher correlation coefficients, which may be mainly attributed to the high enrichment factors of these TEs in $\mathrm{PM}_{2.5}{ }^{6}$ Cobalt, chromium, manganese and zinc predictions have the relatively lower correlation coefficients in April. Cadmium predictions have the lowest correlation coefficients in all months, ranging from 0.4 to 0.58 . TEs simulations show errors to different extent, which may be mainly due to the bias in meteorological simulations, uncertainties in emission estimations and the lack of some pollution sources such as road dust and construction dust emissions in China. ${ }^{7}$ In general, the model could basically reproduce the time trend and magnitude of most of the TEs concentrations. 
The observational TEs concentrations in Chengde (CD), Shijiazhuang (SJZ), Tianjin (TJ), and Langfang (LF) (North China); Shenyang (SY) and Dalian (DL) (Northeast China), Nanjing (NJ), Shanghai (SH), Hangzhou (HZ) and Jinan (JN) (East China); Hengyang (HY), Changsha (CS), Shenzhen (SZ), Guangzhou (GZ) and Nanning (NN) (Central and South China); Guiyang (GY) (Southwest China); Lanzhou (LZ) and Xi' an (XA) (Northwest China) in 2012 and 2015 are collected from other related literatures. ${ }^{8-23}$ Due to the discrepancy and availability of observational data for various TEs reported in previous literatures, only the annual or monthly mean concentrations of cadmium, arsenic, lead and zinc observations are collected in this study to conduct the model evaluation. Figure S5 and S6 display the comparisons between annual or monthly mean simulated and observed concentrations of cadmium, arsenic, lead and zinc for different typical cities in 2012 and 2015, respectively. As can be seen, the NMBs are all negative for all species in these cities. The model has relatively worse ability to reproduce cadmium concentrations, with the NMBs of about $-124 \%$ and 156\% in 2012 and 2015, respectively. Arsenic, lead and zinc predictions have relatively lower NMBs, which are all within $-50 \%$ in 2015 , satisfying the criteria proposed. The NMBs for arsenic and zinc is slightly higher than $-50 \%$ in $2012(-51 \%$ and $-64 \%)$. Correlation coefficients of these simulations compared with the corresponding observations in 2012 and 2015 ranged from 0.87 to 0.67. Arsenic, lead and zinc predictions have relatively higher correlation coefficients (higher than 0.7). The NMEs for arsenic, lead and zinc are basically within or slightly higher than $50 \%$. Cadmium also has the relatively higher NMEs. The under-prediction of all TEs concentrations are found in all cities, especially for cadmium. These under-predictions may be closely related with the lack of some pollution sources such as road dust and construction dust emissions, as well as the over-estimations of wind speeds. ${ }^{7,24}$

Comparisons of spatial variation characteristics between simulations and surface observations are also very useful information for model performance evaluation. Due to the lack of routinely available surface observed concentrations of trace elements in China and the lack of surface $\mathrm{PM}_{2.5}$ observed concentrations in 2012, we collect the surface observed $\mathrm{PM}_{2.5}$ concentrations in 366 cities of China for the year of 2015 from China National Environmental Monitoring Centre (http://beijingair.sinaapp.com). Figure S7 displays the comparison of spatial variations between simulated and surface observed $\mathrm{PM}_{2.5}$ concentrations for the year of 2015 in mainland China. As can be seen in Figure S7 (a), our model well reproduces the spatial variations of $\mathrm{PM}_{2.5}$ concentrations over China, with NMB and NME being $-1.1 \%$ and 30.1\%, respectively. Figure S7 (b) presents the correlation coefficients between simulated and surface observed daily $\mathrm{PM}_{2.5}$ concentrations in each city in 2015. Of the 366 cities compared, 236 cities have correlation coefficients higher than 0.6 , which indicates that our simulations could well capture the spatial variations of $\mathrm{PM}_{2.5}$ concentrations. Overall, our simulation are reasonable and acceptable, and could successfully reproduce the spatiotemporal variations of atmospheric $\mathrm{PM}_{2.5}$ and TEs over China. 


\section{Supplementary Section 3}

\section{Uncertainty analysis}

A Monte Carlo simulation is used to access uncertainty based on the uncertainties of atmospheric TEs emission inventory. Details about the probability distribution for each emission source applied in this study are listed in Table S9.25-27 Emissions of 11 TEs from primary anthropogenic sources with uncertainties in 2015 are summarized in Table S10 and Figure S8. The overall uncertainties of the total emissions in our inventories quantified by the Monte Carlo simulation are $-29.5 \%$ to $59.1 \%$. Among all the emission sources, uncertainties for coal combustion sectors especially for power plants emissions are smallest. This may mainly be attributed to the relatively more detailed activity data and emission control devices in this subcategories. Nonmetallic mineral manufacturing and non-ferrous metal smelting emissions also had a relatively smaller uncertainty, which may be mainly due to the complete official statistics database and their simple production process. In contrast, higher uncertainties of TEs emissions are found in non-coal combustion categories, particular for biomass combustion and brake and tire wear emissions. These high uncertainties of TEs emissions can be mainly from the incomplete of knowledge emission factors and activity data, as well as the absence of adequate field test data in China. Generally speaking, the comprehensive and high resolution emission inventory established in this study could well reflect the real emission situation of 11 TEs in China and can provide reliable input data for the air quality modeling system and support for the policy making. 


\section{Supplementary Section 4}

\section{Non-carcinogenic health risk assessment}

As can be seen in Figure S25, the regions with higher HI values appear in northern China and eastern China. Moreover, it is obvious that compared to adults, the HI values exceeding the threshold for children span a much wider areas and higher values, mainly due to the fact that children are more likely to have higher rates of TEs absorption due to the unique physiological characteristics. The number of people, living in areas with HI exceeding the threshold, is displayed in Figure S26. The results show that the number of people lived in the environment with HI exceeding 1 from 2012 to 2015 has been abated from 55.6 million to 5.7 million, among which, 10.3 million and 12.2 million reductions are found in northern China and eastern China, respectively. However, people lived in Shandong, Hebei provinces are more likely to be affected by non-carcinogenic risks from TEs exposure in 2015, which indicates that the non-carcinogenic risks of TEs still need to be addressed. 
Table S1. Summary of trace element species and the associated emission source categories

\begin{tabular}{|c|c|c|c|c|c|c|c|c|c|c|c|c|}
\hline Sector & Category & $\mathrm{Cd}$ & Co & $\mathrm{Sb}$ & As & $\mathrm{Se}$ & $\mathrm{Ni}$ & $\mathrm{Cr}$ & $\mathrm{Cu}$ & $\mathrm{Mn}$ & $\mathrm{Pb}$ & $\mathrm{Zn}$ \\
\hline \multirow{4}{*}{ Power plant } & Raw coal & $\sqrt{ }$ & $\sqrt{ }$ & $\sqrt{ }$ & $\sqrt{ }$ & $\sqrt{ }$ & $\sqrt{ }$ & $\sqrt{ }$ & $\sqrt{ }$ & $\sqrt{ }$ & $\sqrt{ }$ & $\sqrt{ }$ \\
\hline & Cleaned coal & $\sqrt{ }$ & $\sqrt{ }$ & $\sqrt{ }$ & $\sqrt{ }$ & $\sqrt{ }$ & $\sqrt{ }$ & $\sqrt{ }$ & $\sqrt{ }$ & $\sqrt{ }$ & $\sqrt{ }$ & $\sqrt{ }$ \\
\hline & Briquette & $\sqrt{ }$ & $\sqrt{ }$ & $\sqrt{ }$ & $\sqrt{ }$ & $\sqrt{ }$ & $\sqrt{ }$ & $\sqrt{ }$ & $\sqrt{ }$ & $\sqrt{ }$ & $\sqrt{ }$ & $\sqrt{ }$ \\
\hline & Coke & $\sqrt{ }$ & $\sqrt{ }$ & $\sqrt{ }$ & $\sqrt{ }$ & $\sqrt{ }$ & $\sqrt{ }$ & $\sqrt{ }$ & $\sqrt{ }$ & $\sqrt{ }$ & $\sqrt{ }$ & $\sqrt{ }$ \\
\hline \multirow{4}{*}{ Industrial sector } & Raw coal & $\sqrt{ }$ & $\sqrt{ }$ & $\sqrt{ }$ & $\sqrt{ }$ & $\sqrt{ }$ & $\sqrt{ }$ & $\sqrt{ }$ & $\sqrt{ }$ & $\sqrt{ }$ & $\sqrt{ }$ & $\sqrt{ }$ \\
\hline & Cleaned coal & $\sqrt{ }$ & $\sqrt{ }$ & $\sqrt{ }$ & $\sqrt{ }$ & $\sqrt{ }$ & $\sqrt{ }$ & $\sqrt{ }$ & $\sqrt{ }$ & $\sqrt{ }$ & $\sqrt{ }$ & $\sqrt{ }$ \\
\hline & Briquette & $\sqrt{ }$ & $\sqrt{ }$ & $\sqrt{ }$ & $\sqrt{ }$ & $\sqrt{ }$ & $\sqrt{ }$ & $\sqrt{ }$ & $\sqrt{ }$ & $\sqrt{ }$ & $\sqrt{ }$ & $\sqrt{ }$ \\
\hline & Coke & $\sqrt{ }$ & $\sqrt{ }$ & $\sqrt{ }$ & $\sqrt{ }$ & $\sqrt{ }$ & $\sqrt{ }$ & $\sqrt{ }$ & $\sqrt{ }$ & $\sqrt{ }$ & $\sqrt{ }$ & $\sqrt{ }$ \\
\hline \multirow{4}{*}{ Residential sector } & Raw coal & $\sqrt{ }$ & $\sqrt{ }$ & $\sqrt{ }$ & $\sqrt{ }$ & $\sqrt{ }$ & $\sqrt{ }$ & $\sqrt{ }$ & $\sqrt{ }$ & $\sqrt{ }$ & $\sqrt{ }$ & $\sqrt{ }$ \\
\hline & Cleaned coal & $\sqrt{ }$ & $\sqrt{ }$ & $\sqrt{ }$ & $\sqrt{ }$ & $\sqrt{ }$ & $\sqrt{ }$ & $\sqrt{ }$ & $\sqrt{ }$ & $\sqrt{ }$ & $\sqrt{ }$ & $\sqrt{ }$ \\
\hline & Briquette & $\sqrt{ }$ & $\sqrt{ }$ & $\sqrt{ }$ & $\sqrt{ }$ & $\sqrt{ }$ & $\sqrt{ }$ & $\sqrt{ }$ & $\sqrt{ }$ & $\sqrt{ }$ & $\sqrt{ }$ & $\sqrt{ }$ \\
\hline & Coke & $\sqrt{ }$ & $\sqrt{ }$ & $\sqrt{ }$ & $\sqrt{ }$ & $\sqrt{ }$ & $\sqrt{ }$ & $\sqrt{ }$ & $\sqrt{ }$ & $\sqrt{ }$ & $\sqrt{ }$ & $\sqrt{ }$ \\
\hline \multirow{4}{*}{ Other sector } & Raw coal & $\sqrt{ }$ & $\sqrt{ }$ & $\sqrt{ }$ & $\sqrt{ }$ & $\sqrt{ }$ & $\sqrt{ }$ & $\sqrt{ }$ & $\sqrt{ }$ & $\sqrt{ }$ & $\sqrt{ }$ & $\sqrt{ }$ \\
\hline & Cleaned coal & $\sqrt{ }$ & $\sqrt{ }$ & $\sqrt{ }$ & $\sqrt{ }$ & $\sqrt{ }$ & $\sqrt{ }$ & $\sqrt{ }$ & $\sqrt{ }$ & $\sqrt{ }$ & $\sqrt{ }$ & $\sqrt{ }$ \\
\hline & Briquette & $\sqrt{ }$ & $\sqrt{ }$ & $\sqrt{ }$ & $\sqrt{ }$ & $\sqrt{ }$ & $\sqrt{ }$ & $\sqrt{ }$ & $\sqrt{ }$ & $\sqrt{ }$ & $\sqrt{ }$ & $\sqrt{ }$ \\
\hline & Coke & $\sqrt{ }$ & $\sqrt{ }$ & $\sqrt{ }$ & $\sqrt{ }$ & $\sqrt{ }$ & $\sqrt{ }$ & $\sqrt{ }$ & $\sqrt{ }$ & $\sqrt{ }$ & $\sqrt{ }$ & $\sqrt{ }$ \\
\hline \multirow{7}{*}{ Liquid fuel combustion } & Crude oil & $\sqrt{ }$ & $\sqrt{ }$ & & $\sqrt{ }$ & $\sqrt{ }$ & $\sqrt{ }$ & $\sqrt{ }$ & $\sqrt{ }$ & $\sqrt{ }$ & $\sqrt{ }$ & $\sqrt{ }$ \\
\hline & Gasoline & $\sqrt{ }$ & $\sqrt{ }$ & & $\sqrt{ }$ & $\sqrt{ }$ & $\sqrt{ }$ & $\sqrt{ }$ & $\sqrt{ }$ & $\sqrt{ }$ & $\sqrt{ }$ & $\sqrt{ }$ \\
\hline & Diesel for stationary sources & $\sqrt{ }$ & $\sqrt{ }$ & & $\sqrt{ }$ & $\sqrt{ }$ & $\sqrt{ }$ & $\sqrt{ }$ & $\sqrt{ }$ & $\sqrt{ }$ & $\sqrt{ }$ & $\sqrt{ }$ \\
\hline & Diesel for transportation & $\sqrt{ }$ & $\sqrt{ }$ & & $\sqrt{ }$ & $\sqrt{ }$ & $\sqrt{ }$ & $\sqrt{ }$ & $\sqrt{ }$ & $\sqrt{ }$ & $\sqrt{ }$ & $\sqrt{ }$ \\
\hline & Fuel oil & $\sqrt{ }$ & $\sqrt{ }$ & & $\sqrt{ }$ & $\sqrt{ }$ & $\sqrt{ }$ & $\sqrt{ }$ & $\sqrt{ }$ & $\sqrt{ }$ & $\sqrt{ }$ & $\sqrt{ }$ \\
\hline & $\begin{array}{l}\text { Kerosene for stationary } \\
\text { sources }\end{array}$ & $\sqrt{ }$ & $\sqrt{ }$ & & $\sqrt{ }$ & $\sqrt{ }$ & $\sqrt{ }$ & $\sqrt{ }$ & $\sqrt{ }$ & $\sqrt{ }$ & $\sqrt{ }$ & $\sqrt{ }$ \\
\hline & Kerosene for transportation & $\sqrt{ }$ & $\sqrt{ }$ & & & $\sqrt{ }$ & $\sqrt{ }$ & $\sqrt{ }$ & $\sqrt{ }$ & $\sqrt{ }$ & $\sqrt{ }$ & $\sqrt{ }$ \\
\hline \multirow{2}{*}{ Ferrous metal smelting } & Pig iron & $\sqrt{ }$ & & $\sqrt{ }$ & $\sqrt{ }$ & $\sqrt{ }$ & $\sqrt{ }$ & $\sqrt{ }$ & $\sqrt{ }$ & $\sqrt{ }$ & $\sqrt{ }$ & $\sqrt{ }$ \\
\hline & Steel & $\sqrt{ }$ & & $\sqrt{ }$ & $\sqrt{ }$ & $\sqrt{ }$ & $\sqrt{ }$ & $\sqrt{ }$ & $\sqrt{ }$ & $\sqrt{ }$ & $\sqrt{ }$ & $\sqrt{ }$ \\
\hline \multirow{10}{*}{$\begin{array}{l}\text { Nonferrous metal } \\
\text { smelting }\end{array}$} & Primary copper & $\sqrt{ }$ & & $\sqrt{ }$ & $\sqrt{ }$ & $\sqrt{ }$ & $\sqrt{ }$ & $\sqrt{ }$ & $\sqrt{ }$ & $\sqrt{ }$ & $\sqrt{ }$ & $\sqrt{ }$ \\
\hline & Secondary copper & $\sqrt{ }$ & & $\sqrt{ }$ & $\sqrt{ }$ & $\sqrt{ }$ & $\sqrt{ }$ & $\sqrt{ }$ & $\sqrt{ }$ & & $\sqrt{ }$ & $\sqrt{ }$ \\
\hline & Primary lead & $\sqrt{ }$ & & $\sqrt{ }$ & $\sqrt{ }$ & $\sqrt{ }$ & $\sqrt{ }$ & $\sqrt{ }$ & $\sqrt{ }$ & & $\sqrt{ }$ & $\sqrt{ }$ \\
\hline & Secondary lead & $\sqrt{ }$ & & & $\sqrt{ }$ & & & $\sqrt{ }$ & $\sqrt{ }$ & & $\sqrt{ }$ & $\sqrt{ }$ \\
\hline & Primary zinc & $\sqrt{ }$ & & $\sqrt{ }$ & $\sqrt{ }$ & $\sqrt{ }$ & $\sqrt{ }$ & $\sqrt{ }$ & $\sqrt{ }$ & & $\sqrt{ }$ & $\sqrt{ }$ \\
\hline & Secondary zinc & $\sqrt{ }$ & & & $\sqrt{ }$ & & & $\sqrt{ }$ & & & $\sqrt{ }$ & $\sqrt{ }$ \\
\hline & Primary aluminum & $\sqrt{ }$ & & & & & $\sqrt{ }$ & $\sqrt{ }$ & & & & $\sqrt{ }$ \\
\hline & Secondary aluminum & $\sqrt{ }$ & & & $\sqrt{ }$ & & $\sqrt{ }$ & $\sqrt{ }$ & $\sqrt{ }$ & $\sqrt{ }$ & $\sqrt{ }$ & $\sqrt{ }$ \\
\hline & Nickel & & & & & & $\sqrt{ }$ & & & & & \\
\hline & Antimony & & & $\sqrt{ }$ & & & & & & & & \\
\hline \multirow{3}{*}{$\begin{array}{l}\text { Non-metallic minerals } \\
\text { manufacturing }\end{array}$} & Cement & $\sqrt{ }$ & & & $\sqrt{ }$ & $\sqrt{ }$ & $\sqrt{ }$ & $\sqrt{ }$ & $\sqrt{ }$ & $\sqrt{ }$ & $\sqrt{ }$ & $\sqrt{ }$ \\
\hline & Glass & $\sqrt{ }$ & & & $\sqrt{ }$ & $\sqrt{ }$ & $\sqrt{ }$ & $\sqrt{ }$ & $\sqrt{ }$ & & $\sqrt{ }$ & $\sqrt{ }$ \\
\hline & Brick & $\sqrt{ }$ & $\sqrt{ }$ & $\sqrt{ }$ & $\sqrt{ }$ & $\sqrt{ }$ & $\sqrt{ }$ & $\sqrt{ }$ & & $\sqrt{ }$ & $\sqrt{ }$ & \\
\hline $\begin{array}{l}\text { Municipal solid waste } \\
\text { (MSW) incineration }\end{array}$ & Municipal solid waste & $\sqrt{ }$ & $\sqrt{ }$ & $\sqrt{ }$ & $\sqrt{ }$ & $\sqrt{ }$ & $\sqrt{ }$ & $\sqrt{ }$ & $\sqrt{ }$ & $\sqrt{ }$ & $\sqrt{ }$ & $\sqrt{ }$ \\
\hline \multirow{2}{*}{ Biomass burning } & Straw & $\sqrt{ }$ & $\sqrt{ }$ & $\sqrt{ }$ & $\sqrt{ }$ & $\sqrt{ }$ & $\sqrt{ }$ & $\sqrt{ }$ & $\sqrt{ }$ & $\sqrt{ }$ & $\sqrt{ }$ & $\sqrt{ }$ \\
\hline & Wood & $\sqrt{ }$ & $\sqrt{ }$ & $\sqrt{ }$ & $\sqrt{ }$ & $\sqrt{ }$ & $\sqrt{ }$ & $\sqrt{ }$ & $\sqrt{ }$ & $\sqrt{ }$ & $\sqrt{ }$ & $\sqrt{ }$ \\
\hline \multirow{2}{*}{$\begin{array}{l}\text { Brake and Tire (B\&TW) } \\
\text { wear }\end{array}$} & Brake pad & $\sqrt{ }$ & $\sqrt{ }$ & $\sqrt{ }$ & $\sqrt{ }$ & $\sqrt{ }$ & $\sqrt{ }$ & $\sqrt{ }$ & $\sqrt{ }$ & $\sqrt{ }$ & $\sqrt{ }$ & $\sqrt{ }$ \\
\hline & Tire & $\sqrt{ }$ & $\sqrt{ }$ & $\sqrt{ }$ & $\sqrt{ }$ & $\sqrt{ }$ & $\sqrt{ }$ & $\sqrt{ }$ & $\sqrt{ }$ & $\sqrt{ }$ & $\sqrt{ }$ & $\sqrt{ }$ \\
\hline
\end{tabular}


Table S2. Data sources of activity data for the trace element emission sectors in China

\begin{tabular}{|c|c|}
\hline Emission sectors & Data Sources \\
\hline \multirow{3}{*}{ Coal consumption by power plants } & China Electric Power Yearbook \\
\hline & China Editorial Power Industry Statistics \\
\hline & China Energy Statistical Yearbook \\
\hline \multirow{3}{*}{ Coal consumption by industrial boilers } & China Industry Statistical Yearbook \\
\hline & China Coal Industry Yearbook \\
\hline & China Energy Statistical Yearbook \\
\hline Coal consumption by residential sectors & China Energy Statistical Yearbook \\
\hline Coal consumption by other sectors & China Energy Statistical Yearbook \\
\hline \multirow{2}{*}{ Biomass burning } & China Statistical Yearbook \\
\hline & China Rural Statistical Yearbook \\
\hline Liquid fuels combustion & China Energy Statistical Yearbook \\
\hline Nonferrous metals smelting & $\begin{array}{l}\text { The Yearbook of Nonferrous Metals Industry of } \\
\text { China } \\
\text { China Statistical Yearbook }\end{array}$ \\
\hline Non-metallic minerals manufacturing & $\begin{array}{l}\text { China Cement Almanac } \\
\text { China Statistical Yearbook } \\
\text { China Steel Yearbook }\end{array}$ \\
\hline Ferrous metal smelting & $\begin{array}{l}\text { China Statistical Yearbook } \\
\text { China Industry Statistical Yearbook }\end{array}$ \\
\hline Municipal solid waste incineration & $\begin{array}{l}\text { Statistical Yearbook of Urban Construction of China } \\
\text { China Statistical Yearbook }\end{array}$ \\
\hline Brake and tire wear & $\begin{array}{l}\text { China Automotive Industry Yearbook } \\
\text { China's Auto Market Almanac } \\
\text { China Statistical Yearbook }\end{array}$ \\
\hline
\end{tabular}


Table S3. Spatial allocation method of TEs emission for each source

\begin{tabular}{cll}
\hline & Source category & \multicolumn{1}{c}{ Spatial surrogates } \\
\hline \multirow{4}{*}{ Coal combustion } & Coal combustion by power plants & Latitudes and longitudes \\
& Coal combustion by industrial Boilers & Industrial gross domestic product \\
& Coal combustion by residential sector & Population density \\
& Coal combustion by other sector & Gross Domestic Product \\
& Liquid fuel combustion & Industrial gross domestic product \\
& Ferrous metal smelting & Industrial gross domestic product \\
Non-coal & Nonferrous metal smelting & Industrial gross domestic product \\
combustion & Non-metallic minerals manufacturing & Industrial gross domestic product \\
& Municipal solid waste incineration & Population density \\
& Biomass burning & Land use type \\
& Brake and Tyre wear & Road network /population \\
\end{tabular}


Table S4. Comparison of meteorological predictions vs. observations over China, 2012

\begin{tabular}{|c|c|c|c|c|c|c|c|c|c|c|c|c|c|c|}
\hline & & Jan & Feb & Mar & Apr & May & Jun & Jul & Aug & Sep & Oct & Nov & Dec I & ench-mark \\
\hline \multirow{4}{*}{$\mathrm{T} 2(\mathrm{~K})$} & NMB (\%) & -33.3 & -30.2 & -33.1 & -33.7 & -28.3 & -29.3 & -29.7 & -36.1 & -32.1 & -28.6 . & 37.1 & 31.9 & \multirow{4}{*}{$\leq \pm 0.5$} \\
\hline & NME (\%) & 49.6 & 38.7 & 22.3 & 28.8 & 27.6 & 19.6 & 24.3 & 29.5 & 28.9 & 38.5 & 47.4 & 37.3 & \\
\hline & $\mathrm{MB}$ & -0.9 & -1.3 & -1.2 & -0.7 & -0.6 & -0.4 & -0.6 & -0.7 & -0.6 & -0.5 & 0.4 & 0.5 & \\
\hline & RMSE & 3.9 & 3.5 & 4.1 & 3.3 & 3.2 & 3.3 & 3.6 & 3.5 & 3.4 & 3.5 & 3.7 & 3.3 & \\
\hline \multirow{4}{*}{$\begin{array}{c}\text { WS10 } \\
(\mathrm{m} / \mathrm{s})\end{array}$} & NMB (\%) & 35.6 & 23.2 & 27.1 & 38.1 & 32.3 & 31.2 & 24.3 & 26.2 & 28.3 & 24.2 & 28.1 & 36.2 & \multirow{4}{*}{$\begin{array}{c}\leq \pm 0.5 \\
\leq 2.0\end{array}$} \\
\hline & NME (\%) & 32.1 & 34.1 & 46.7 & 32.4 & 34.6 & 33.2 & 26.2 & 37.8 & 31.4 & 32.4 & 31.2 & 42.1 & \\
\hline & MB & 0.3 & 0.8 & 0.2 & 1.0 & 0.7 & 0.5 & 0.5 & 0.4 & 0.5 & 0.6 & 1.0 & 1.1 & \\
\hline & RMSE & 2.3 & 2.2 & 2.9 & 2.9 & 2.7 & 2.3 & 2.2 & 1.5 & 2.6 & 2.7 & 2.9 & 2.9 & \\
\hline \multirow{4}{*}{ WD10() } & NMB (\%) & 38.5 & 30.2 & 35.1 & 32.4 & 36.3 & 37.2 & 38.6 & 49.6 & -33.8 & -42.3 & 35.1 & 29.4 & \multirow{4}{*}{$\leq \pm 10$} \\
\hline & NME (\%) & 38.9 & 36.1 & 39.4 & 35.8 & 39.5 & 47.8 & 48.8 & 32.4 & 37.4 & 38.1 & 22.5 & 34.2 & \\
\hline & $\mathrm{MB}$ & 9.5 & 13.1 & 10.4 & 12.4 & 10.2 & 3.6 & 10.8 & 11.9 & -5.4 & -0.9 & 18.5 & 12.7 & \\
\hline & RMSE & 62.3 & 60.5 & 63.2 & 64.8 & 63.8 & 62.6 & 64.5 & 64.7 & 66.7 & 62.3 & 62 & 64.1 & \\
\hline \multirow{4}{*}{ RH (\%) } & NMB (\%) & -37.9 & 36.9 & 47.5 & 29.1 & -35.3 & -39.7 & 31.8 & 34.4 & 39.3 & -40.7 & 32.2 & 23.3 & \\
\hline & NME (\%) & 25.6 & 28.4 & 48.7 & 38.3 & 34.5 & 38.1 & 32.8 & 46.1 & 20.2 & 31.2 & 34.9 & 35.7 & \\
\hline & $\mathrm{MB}$ & -1.6 & 4.6 & 1.1 & 1.8 & -0.3 & -0.5 & 1.2 & 1.7 & 1.6 & -0.7 & 3.3 & -6.5 & \\
\hline & RMSE & 21.7 & 15.3 & 18.4 & 16.9 & 17.5 & 15.4 & 14.3 & 17.6 & 18.3 & 18.4 & 18.1 & 19.1 & \\
\hline
\end{tabular}


Table S5. Comparison of meteorological predictions vs. observations over China, 2015

\begin{tabular}{llccccccccccccc}
\hline & & Jan & Feb & Mar & Apr & May & Jun & Jul & Aug & Sep & Oct & Nov & Dec & Bench-mark \\
\hline \multirow{6}{*}{ T2(K) } & NMB (\%) & -34.9 & -39.3 & -39.3 & -28.7 & -39.6 & -30.2 & -30.6 & -30.7 & -29.9 & 29.1 & 28.1 & 27.7 & \\
& NME (\%) & 33.2 & 26.2 & 27.5 & 28.6 & 29.5 & 32.5 & 33.7 & 36.7 & 30.1 & 29.6 & 28.7 & 37.1 & \\
& MB & -1.1 & -1.3 & -1.2 & -0.9 & -0.6 & -0.3 & -0.5 & -0.5 & -0.4 & 0.2 & 0.2 & 0.1 & $\leq \pm 0.5$ \\
& RMSE & 4.1 & 3.5 & 3.4 & 3.1 & 3.5 & 3.3 & 3.2 & 3.5 & 3.3 & 3.6 & 3.6 & 3.2 & \\
\hline \multirow{6}{*}{ WS } & NMB (\%) & 30.3 & 28.3 & 30.5 & 30.8 & 29.1 & 26.7 & 27.5 & 25.9 & 26.7 & 27.5 & 28.3 & 32.3 & \\
(m/s) & NME (\%) & 34.5 & 39.3 & 42.3 & 36.3 & 32.8 & 28.7 & 33.2 & 28.7 & 30.2 & 33.2 & 34.8 & 35.5 & \\
& MB & 0.2 & 1.1 & 0.9 & 1.1 & 0.9 & 0.5 & 0.5 & 0.6 & 0.8 & 1.2 & 1 & 1.1 & $\leq \pm 0.5$ \\
& RMSE & 2.5 & 2.4 & 2.3 & 2.2 & 2.1 & 1.9 & 2 & 1.7 & 2.1 & 2.1 & 2.5 & 2.2 & $\leq 2.0$ \\
\hline \multirow{5}{*}{ WD(०) } & NMB (\%) & 36.7 & -41.1 & -34.1 & -45.5 & -46.5 & -30.4 & -36.2 & -39.8 & -44.9 & -32.2 & -35.1 & -35.4 & \\
& NME (\%) & 29.1 & 36.4 & 28.5 & 39.5 & 36.8 & 28.4 & 20.9 & 23.2 & 34.4 & 34.6 & 33.3 & 48.5 & \\
& MB & 9.3 & -14.1 & -13.4 & -8.4 & -7.2 & -10.6 & -8.3 & -11.9 & -3.4 & -0.9 & -6.5 & -7.7 & $\leq \pm 10$ \\
& RMSE & 67.3 & 69.1 & 65.1 & 60.1 & 63.6 & 61.4 & 67.5 & 62.4 & 65.3 & 63.9 & 62.8 & 60.8 & \\
\hline \multirow{2}{*}{ RH (\%) } & NMB (\%) & 34.6 & -38.5 & 39.1 & 36.8 & 34.0 & 38.3 & 30.4 & 25.7 & 28.9 & 31.3 & -31.8 & -34.9 & \\
& NME (\%) & 35.6 & 35.7 & 30.6 & 37.4 & 36.0 & 40.3 & 34.3 & 24.4 & 25.4 & 33.2 & 21.1 & 30.9 & \\
& MB & 1.2 & -3.4 & 1.2 & 1.3 & 1.8 & 0.8 & 1.3 & 2.3 & 0.9 & 1.5 & -0.3 & -4.3 & \\
& RMSE & 22.2 & 19.8 & 17.8 & 19.2 & 15.7 & 17.9 & 18.6 & 19.2 & 17.8 & 16.3 & 17.8 & 18.9 &
\end{tabular}


Table S6 Model performance on $\mathrm{PM}_{2.5}$ and trace elements in January, April, August and October over Beijing, 2015

\begin{tabular}{|c|c|c|c|c|c|c|c|c|c|c|c|c|c|c|c|c|}
\hline \multirow{2}{*}{$\begin{array}{l}\text { Species } \\
\left(\mathrm{ng} / \mathrm{m}^{3}\right)\end{array}$} & \multicolumn{4}{|c|}{ Jan. } & \multicolumn{4}{|c|}{ Apr. } & \multicolumn{4}{|c|}{ Aug. } & \multicolumn{4}{|c|}{ Oct. } \\
\hline & MB & $\begin{array}{c}\text { NMB } \\
(\%)\end{array}$ & $\begin{array}{c}\text { NME } \\
(\%)\end{array}$ & $\mathrm{R}$ & MB & $\begin{array}{c}\text { NMB } \\
(\%)\end{array}$ & $\begin{array}{c}\text { NME } \\
(\%)\end{array}$ & $\mathrm{R}$ & MB & $\begin{array}{c}\text { NMB } \\
(\%)\end{array}$ & $\begin{array}{c}\text { NME } \\
(\%)\end{array}$ & $\mathrm{R}$ & MB & $\begin{array}{c}\text { NMB } \\
(\%)\end{array}$ & $\begin{array}{c}\text { NME } \\
(\%)\end{array}$ & $\mathrm{R}$ \\
\hline $\mathrm{PM}_{2.5}\left(\mathrm{ug} / \mathrm{m}^{3}\right)$ & -13.6 & -45 & 46 & 0.9 & -18 & -32 & 41 & 0.9 & -7.8 & -21 & 30 & 0.7 & -14 & -21.8 & 25 & 0.89 \\
\hline $\mathrm{Cd}$ & -1.6 & -181 & 184 & 0.5 & -1.2 & -173 & 173 & 0.6 & -0.2 & -62 & 71 & 0.4 & -0.8 & -146 & 161 & 0.4 \\
\hline $\mathrm{Co}$ & -0.3 & -28 & 44 & 0.7 & -0.4 & -51 & 57 & 0.8 & -0.1 & -58 & 66 & 0.4 & -0.1 & -15 & 46 & 0.49 \\
\hline $\mathrm{Sb}$ & -2.9 & -72 & 77 & 0.7 & -5.2 & -44 & 47 & 0.8 & -2.4 & -56 & 62 & 0.6 & -2.4 & -50 & 69 & 0.7 \\
\hline As & -4.3 & -54 & 62 & 0.8 & -4.8 & -63 & 63 & 0.9 & -0.9 & -31 & 50 & 0.6 & -3.1 & -54 & 66 & 0.82 \\
\hline $\mathrm{Se}$ & -1.2 & -18 & 37 & 0.8 & -1.9 & -57 & 60 & 0.6 & -1.0 & -28 & 46 & 0.6 & -1.1 & -39 & 69 & 0.71 \\
\hline $\mathrm{Ni}$ & -1.0 & -25 & 73 & 0.6 & -5.7 & -32 & 32 & 0.7 & -1.4 & -44 & 45 & 0.7 & -5.9 & -68 & 68 & 0.73 \\
\hline $\mathrm{Cr}$ & -8.3 & -60 & 79 & 0.6 & -22 & -62 & 62 & 0.5 & -6.6 & -76 & 78 & 0.7 & -6.7 & -36 & 37 & 0.6 \\
\hline $\mathrm{Cu}$ & -6.8 & -49 & 51 & 0.9 & -5.4 & -41 & 41 & 0.7 & -5.6 & -54 & 57 & 0.7 & -2.3 & -54 & 57 & 0.86 \\
\hline $\mathrm{Mn}$ & -2.8 & -55 & 58 & 0.9 & -2.9 & -78 & 78 & 0.5 & -1.3 & -58 & 58 & 0.8 & -2.3 & -77 & 91 & 0.77 \\
\hline $\mathrm{Pb}$ & -55.8 & -67 & 75 & 0.8 & -53 & -68 & 68 & 0.8 & -11 & -47 & 62 & 0.7 & -17 & -49 & 65 & 0.83 \\
\hline $\mathrm{Zn}$ & -68.4 & -53 & 89 & 0.8 & -62 & -39 & 47 & 0.6 & -15 & -65 & 74 & 0.8 & -57 & -64 & 65 & 0.92 \\
\hline
\end{tabular}


Table S7. Infiltration factors and annual mean amount of time indoors and outdoors

\begin{tabular}{|c|c|c|c|}
\hline Province & $F_{\text {inf }}$ & $t_{\text {out }}$ & $\mathrm{t}_{\text {in }}$ \\
\hline Beijing & 0.58 & 0.13 & 0.87 \\
\hline Tianjin & 0.53 & 0.13 & 0.88 \\
\hline Hebei & 0.51 & 0.11 & 0.89 \\
\hline Shanxi & 0.51 & 0.12 & 0.88 \\
\hline Inner Mongolia & 0.48 & 0.13 & 0.88 \\
\hline Liaoning & 0.51 & 0.1 & 0.9 \\
\hline Jilin & 0.48 & 0.09 & 0.91 \\
\hline Heilongjiang & 0.5 & 0.09 & 0.91 \\
\hline Shanghai & 0.74 & 0.11 & 0.89 \\
\hline Jiangsu & 0.72 & 0.1 & 0.9 \\
\hline Zhejiang & 0.76 & 0.11 & 0.89 \\
\hline Anhui & 0.71 & 0.11 & 0.89 \\
\hline Fujian & 0.81 & 0.12 & 0.88 \\
\hline Jiangxi & 0.82 & 0.14 & 0.86 \\
\hline Shandong & 0.57 & 0.09 & 0.91 \\
\hline Henan & 0.59 & 0.17 & 0.83 \\
\hline Hubei & 0.74 & 0.15 & 0.85 \\
\hline Hunan & 0.76 & 0.12 & 0.88 \\
\hline Guangdong & 0.87 & 0.14 & 0.86 \\
\hline Guangxi & 0.77 & 0.18 & 0.82 \\
\hline Hainan & 0.94 & 0.16 & 0.84 \\
\hline Chongqing & 0.82 & 0.15 & 0.85 \\
\hline Sichuan & 0.76 & 0.17 & 0.83 \\
\hline Guizhou & 0.73 & 0.12 & 0.88 \\
\hline Yunnan & 0.76 & 0.2 & 0.8 \\
\hline Tibet & 0.51 & 0.17 & 0.83 \\
\hline Shaanxi & 0.52 & 0.16 & 0.84 \\
\hline Gansu & 0.51 & 0.21 & 0.79 \\
\hline Qinghai & 0.48 & 0.1 & 0.9 \\
\hline Ningxia & 0.55 & 0.11 & 0.89 \\
\hline Xinjiang & 0.5 & 0.16 & 0.84 \\
\hline
\end{tabular}


Table S8. Reaction parameters for various TEs

\begin{tabular}{lcc}
\hline & $\begin{array}{c}R f D \\
\left(\mathrm{mg} \mathrm{kg}^{-1} \mathrm{day}^{-1}\right)\end{array}$ & $\begin{array}{c}S F \\
\left(\mathrm{mg} \mathrm{kg}^{-1} \mathrm{day}^{-1}\right)^{-1}\end{array}$ \\
\hline $\mathrm{Pb}$ & $3.50 \mathrm{E}-03^{[28],[30]}$ & $4.2 \mathrm{E}-02^{[28],[31]}$ \\
$\mathrm{Zn}$ & $3.00 \mathrm{E}-01^{[28],[29]}$ & $/$ \\
$\mathrm{Cu}$ & $4.02 \mathrm{E}-03^{[28],[30]}$ & $/$ \\
$\mathrm{Mn}$ & $1.40 \mathrm{E}-05^{[28]}$ & $/$ \\
$\mathrm{Se}$ & $5.70 \mathrm{E}-05^{[28],[29]}$ & $/$ \\
$\mathrm{Sb}$ & $1.40 \mathrm{E}-04^{[28],[29]}$ & $2.8 \mathrm{E}-02^{[28],[31]}$ \\
$\mathrm{As}$ & $3.00 \mathrm{E}-04^{[28]}$ & $1.51 \mathrm{E}+01^{[28]}$ \\
$\mathrm{Cd}$ & $1.00 \mathrm{E}-04^{[28]}$ & $6.30 \mathrm{E}+00^{[28]}$ \\
$\mathrm{Cr}$ & $3.00 \mathrm{E}-05^{[28],[29]}$ & $4.20 \mathrm{E}+01(\mathrm{Cr}(\mathrm{VI}))^{[28]}$ \\
$\mathrm{Ni}$ & $2.06 \mathrm{E}-02^{[28],[29]}$ & $9.01 \mathrm{E}-01^{[28]}$ \\
$\mathrm{Co}$ & $5.71 \mathrm{E}-06^{[28]}$ & $9.80 \mathrm{E}+00^{[28]}$ \\
\hline
\end{tabular}


Table S9. Probability distribution for each parameter for uncertainty analysis

\begin{tabular}{|c|c|c|c|}
\hline Categories & Parameter description & Probability distribution & Data sources \\
\hline \multicolumn{4}{|c|}{ Coal combustion sources } \\
\hline \multirow{5}{*}{ Coal consumption } & Power plants & Normal (CV: 5\%) & {$[25]$} \\
\hline & Industrial sectors & Normal (CV: 10\%) & {$[26]$} \\
\hline & Residential sectors & Normal (CV: 14\%) & {$[26]$} \\
\hline & Other sectors & Normal (CV: 16\%) & {$[26]$} \\
\hline & Pulverized-coal boiler & Triangular & Data fitting \\
\hline \multirow{3}{*}{ Release rate } & Stoker fired boiler & Triangular & Data fitting \\
\hline & Fluidized-bed furnace & Triangular & Data fitting \\
\hline & Coke furnace & Triangular & Data fitting \\
\hline \multirow{6}{*}{$\begin{array}{l}\text { Removal } \\
\text { efficiency }\end{array}$} & ESPs & Normal (CV: 5\%) & Subject judgement \\
\hline & EFs & Uniform & Data fitting \\
\hline & Wet scrubber & Triangular & Subject judgement \\
\hline & Cyclone & Normal (CV: 20\%) & Subject judgement \\
\hline & Wet-FGD & Triangular & Subject judgement \\
\hline & Coal washing & Uniform & Data fitting \\
\hline \multicolumn{4}{|c|}{ Non-coal combustion sources } \\
\hline \multirow{4}{*}{ Biomass burning } & Biofuel consumption & Normal (CV: 20\%) & {$[27]$} \\
\hline & Emission factors & Triangular & {$[27]$} \\
\hline & Ratio of biomass burning & $\begin{array}{l}\text { Normal } \\
\text { dependent) }\end{array}$ & {$[27]$} \\
\hline & straw-to-crop ratio & $\begin{array}{l}\text { Uniform } \\
\text { dependent) }\end{array}$ & {$[27]$} \\
\hline \multirow{2}{*}{$\begin{array}{l}\text { Liquid fuel } \\
\text { combustion }\end{array}$} & Liquid fuel consumption & Normal (CV: 5\%) & {$[27]$} \\
\hline & Emission factors & Normal (CV: 25\%) & Subject judgement \\
\hline \multirow{2}{*}{$\begin{array}{l}\text { Nonferrous metal } \\
\text { smelting }\end{array}$} & Nonferrous metal production & Normal (CV: 5\%) & {$[27]$} \\
\hline & Emission factors & Triangular & Data fitting \\
\hline \multirow{3}{*}{$\begin{array}{l}\text { Non-metallic } \\
\text { minerals } \\
\text { manufacturing }\end{array}$} & $\begin{array}{l}\text { Output of Cement/ glass / } \\
\text { brick }\end{array}$ & Normal (CV: 20\%) & Subject judgement \\
\hline & $\begin{array}{l}\text { emission factors (cement, } \\
\text { glass) }\end{array}$ & Normal (CV: 25\%) & Subject judgement \\
\hline & emission factors (brick) & Normal (CV: 30\%) & Subject judgement \\
\hline \multirow{2}{*}{$\begin{array}{l}\text { Ferrous metal } \\
\text { smelting }\end{array}$} & Pig iron and steel yield & Normal (CV: 15\%) & {$[27]$} \\
\hline & Emission factors & Triangular & Subject judgement \\
\hline \multirow{2}{*}{$\begin{array}{l}\text { Municipal solid } \\
\text { waste incineration }\end{array}$} & MSW consumption & Normal (CV: 20\%) & Subject judgement \\
\hline & Emission factors & Normal (CV: 20\%) & Subject judgement \\
\hline \multirow{4}{*}{$\begin{array}{l}\text { Brake and tire } \\
\text { wear }\end{array}$} & Vehicle number & Normal (CV: 5\%) & {$[27]$} \\
\hline & Average vehicle mileage & Normal (CV: 5\%) & {$[27]$} \\
\hline & TSP emission factors & Uniform & Data fitting \\
\hline & Trace element content & Triangular & Data fitting \\
\hline
\end{tabular}


Table S10. Uncertainties in the sectoral emissions of trace elements in China, 2015.

\begin{tabular}{|c|c|c|c|c|c|c|c|c|c|c|c|}
\hline Categories & $\mathrm{Cd}$ & $\mathrm{Co}$ & $\mathrm{Sb}$ & As & $\mathrm{Se}$ & $\mathrm{Ni}$ & $\mathrm{Cr}$ & $\mathrm{Cu}$ & $\mathrm{Mn}$ & $\mathrm{Pb}$ & $\mathrm{Zn}$ \\
\hline ССРP & $-22 \% \sim 28 \%$ & $-35 \% \sim 22 \%$ & $-24 \% \sim 29 \%$ & $-26 \% \sim 22 \%$ & $-25 \% \sim 25 \%$ & $-19 \% \sim 27 \%$ & $-22 \% \sim 24 \%$ & $-26 \% \sim 27 \%$ & $-25 \% \sim 21 \%$ & $-26 \% \sim 29 \%$ & $-23 \% \sim 35 \%$ \\
\hline CCIB & $-43 \% \sim 53 \%$ & $-39 \% \sim 45 \%$ & $-46 \% \sim 46 \%$ & $-44 \% \sim 54 \%$ & $-43 \% \sim 56 \%$ & $-48 \% \sim 53 \%$ & $-69 \% \sim 58 \%$ & $-50 \% \sim 59 \%$ & $-46 \% \sim 41 \%$ & $-55 \% \sim 57 \%$ & $-56 \% \sim 40 \%$ \\
\hline CCRS & $-56 \% \sim 64 \%$ & $-42 \% \sim 44 \%$ & $-58 \% \sim 67 \%$ & $-52 \% \sim 67 \%$ & $-53 \% \sim 67 \%$ & $-52 \% \sim 68 \%$ & $-56 \% \sim 61 \%$ & $-63 \% \sim 50 \%$ & $-58 \% \sim 57 \%$ & $-52 \% \sim 63 \%$ & $-57 \% \sim 50 \%$ \\
\hline $\mathrm{CCOS}$ & $-65 \% \sim 65 \%$ & $-57 \% \sim 62 \%$ & $-61 \% \sim 77 \%$ & $-72 \% \sim 76 \%$ & $-64 \% \sim 79 \%$ & $-8 \% \sim 80 \%$ & $-55 \% \sim 70 \%$ & $-62 \% \sim 74 \%$ & $-66 \% \sim 77 \%$ & $-50 \% \sim 89 \%$ & $-66 \% \sim 62 \%$ \\
\hline $\mathrm{BBS}$ & $-65 \% \sim 65 \%$ & $-75 \% \sim 73 \%$ & $-67 \% \sim 61 \%$ & $-68 \% \sim 66 \%$ & $-72 \% \sim 73 \%$ & $-65 \% \sim 66 \%$ & $-64 \% \sim 69 \%$ & $-74 \% \sim 76 \%$ & $-76 \% \sim 73 \%$ & $-78 \% \sim 64 \%$ & $-74 \% \sim 63 \%$ \\
\hline LFC & $-40 \% \sim 59 \%$ & $-47 \% \sim 51 \%$ & / & $-48 \% \sim 43 \%$ & $-50 \% \sim 53 \%$ & $-52 \% \sim 69 \%$ & $-47 \% \sim 53 \%$ & $-69 \% \sim 52 \%$ & $-54 \% \sim 58 \%$ & $-83 \% \sim 62 \%$ & $-66 \% \sim 44 \%$ \\
\hline NFMS & $-35 \% \sim 36 \%$ & / & $-38 \% \sim 33 \%$ & $-41 \% \sim 46 \%$ & $-35 \% \sim .4 \%$ & $-39 \% \sim 39 \%$ & $-39 \% \sim 33 \%$ & $-58 \% \sim 55 \%$ & $-58 \% \sim 55 \%$ & $36 \% \sim 38 \%$ & $-33 \% \sim 46 \%$ \\
\hline FMS & $-47 \% \sim 57 \%$ & / & $-42 \% \sim 51 \%$ & $-51 \% \sim 67 \%$ & $-42 \% \sim 54 \%$ & $-50 \% \sim 48 \%$ & $-47 \% \sim 45 \%$ & $-47 \% \sim 45 \%$ & $-59 \sim 62 \%$ & $-55 \% \sim 73 \%$ & $-54 \% \sim 49 \%$ \\
\hline NMMM & $-36 \% \sim 45 \%$ & $-43 \% \sim 44 \%$ & $-46 \% \sim 45 \%$ & $-40 \% \sim 45 \%$ & $-41 \% \sim 32 \%$ & $-44 \% \sim 46 \%$ & $-33 \% \sim 36 \%$ & $-37 \% \sim 33 \%$ & $-45 \% \sim 57 \%$ & $-42 \% \sim 47 \%$ & $-44 \% \sim 42 \%$ \\
\hline MSWI & $-66 \% \sim 76 \%$ & $-73 \% \sim 84 \%$ & $-75 \% \sim 71 \%$ & $-63 \% \sim 71 \%$ & $-56 \% \sim 69 \%$ & $-59 \% \sim 70 \%$ & $-58 \% \sim 63 \%$ & $-64 \% \sim 65 \%$ & $-89 \% \sim 69 \%$ & $-63 \% \sim 62 \%$ & $-72 \% \sim 69 \%$ \\
\hline B\&TW & $-74 \% \sim 98 \%$ & $-79 \% \sim 132 \%$ & $-91 \% \sim 137 \%$ & $-89 \% \sim 93 \%$ & $-81 \% \sim 156 \%$ & $-70 \% \sim 84 \%$ & $-90 \% \sim 175 \%$ & $-94 \% \sim 134 \%$ & $-61 \% \sim 68 \%$ & $-80 \% \sim 156 \%$ & $-83 \% \sim 112 \%$ \\
\hline Total & $-34 \% \sim 51 \%$ & $-34 \% \sim 45 \%$ & $-29 \% \sim 50 \%$ & $-30 \% \sim 45 \%$ & $-33 \% \sim 59 \%$ & $-33 \% \sim 43 \%$ & $-31 \% \sim 39 \%$ & $-36 \% \sim 47 \%$ & $-30 \% \sim 49 \%$ & $-31 \% \sim 45 \%$ & $-30 \% \sim 42 \%$ \\
\hline
\end{tabular}


Table S11. Emissions of trace elements in China 2015 by province ( $t)$

\begin{tabular}{|c|c|c|c|c|c|c|c|c|c|c|c|c|c|}
\hline \multirow{2}{*}{ Region } & \multirow{2}{*}{ Province } & \multicolumn{12}{|c|}{ Trace elements } \\
\hline & & $\mathrm{Cd}$ & $\mathrm{Cr}$ & $\mathrm{Pb}$ & As & $\mathrm{Se}$ & $\mathrm{Ni}$ & $\mathrm{Sb}$ & $\mathrm{Mn}$ & $\mathrm{Co}$ & $\mathrm{Cu}$ & $\mathrm{Zn}$ & Total \\
\hline \multirow{5}{*}{$\mathrm{NC}$} & Beijing & 1 & 21 & 71 & 7 & 12 & 10 & 20 & 33 & 2 & 98 & 63 & 338 \\
\hline & Tianjin & 3 & 45 & 119 & 13 & 51 & 33 & 13 & 99 & 5 & 91 & 235 & 706 \\
\hline & Hebei & 16 & 372 & 939 & 158 & 237 & 148 & 58 & 820 & 29 & 569 & 1908 & 5256 \\
\hline & Shanxi & 20 & 203 & 460 & 72 & 137 & 112 & 41 & 414 & 23 & 441 & 1195 & 3120 \\
\hline & Neimenggu & 17 & 157 & 582 & 210 & 73 & 74 & 36 & 1018 & 33 & 348 & 1022 & 3572 \\
\hline \multirow{3}{*}{ NEC } & Liaoning & 13 & 180 & 452 & 66 & 51 & 142 & 35 & 487 & 31 & 299 & 1059 & 2817 \\
\hline & Jilin & 4 & 102 & 243 & 63 & 40 & 51 & 21 & 279 & 19 & 149 & 274 & 1245 \\
\hline & Heilongjiang & 4 & 105 & 291 & 61 & 29 & 53 & 25 & 579 & 29 & 181 & 247 & 1605 \\
\hline \multirow{7}{*}{$\mathrm{EC}$} & Shanghai & 4 & 37 & 120 & 16 & 18 & 77 & 16 & 119 & 5 & 94 & 249 & 757 \\
\hline & Jiangsu & 25 & 267 & 597 & 88 & 150 & 166 & 76 & 493 & 28 & 534 & 1150 & 3576 \\
\hline & Zhejiang & 12 & 155 & 369 & 41 & 107 & 102 & 58 & 294 & 13 & 307 & 588 & 2047 \\
\hline & Anhui & 33 & 223 & 401 & 87 & 151 & 129 & 22 & 190 & 27 & 340 & 598 & 2203 \\
\hline & Fujian & 17 & 96 & 240 & 49 & 79 & 86 & 26 & 167 & 8 & 211 & 357 & 1338 \\
\hline & Jiangxi & 37 & 169 & 423 & 88 & 83 & 101 & 33 & 210 & 9 & 328 & 701 & 2185 \\
\hline & Shandong & 39 & 320 & 838 & 221 & 257 & 251 & 82 & 806 & 33 & 717 & 1352 & 4920 \\
\hline \multirow{6}{*}{$\mathrm{CSC}$} & Henan & 27 & 209 & 679 & 61 & 165 & 118 & 64 & 394 & 21 & 479 & 822 & 3042 \\
\hline & Hubei & 30 & 273 & 587 & 86 & 227 & 131 & 43 & 355 & 24 & 302 & 702 & 2762 \\
\hline & Hunan & 23 & 320 & 710 & 123 & 146 & 101 & 59 & 664 & 26 & 252 & 960 & 3386 \\
\hline & Guangdong & 10 & 176 & 373 & 40 & 133 & 127 & 73 & 300 & 15 & 418 & 554 & 2222 \\
\hline & Guangxi & 25 & 219 & 314 & 70 & 43 & 71 & 34 & 156 & 10 & 236 & 604 & 1782 \\
\hline & Hainan & 1 & 7 & 22 & 2 & 3 & 10 & 4 & 19 & 1 & 17 & 24 & 109 \\
\hline \multirow{4}{*}{ SWC } & Chongqing & 9 & 133 & 231 & 34 & 53 & 56 & 21 & 133 & 23 & 139 & 122 & 955 \\
\hline & Sichuan & 18 & 229 & 394 & 37 & 94 & 88 & 47 & 229 & 20 & 245 & 461 & 1865 \\
\hline & Guizhou & 11 & 239 & 357 & 120 & 105 & 104 & 75 & 581 & 44 & 382 & 401 & 2423 \\
\hline & Yunnan & 37 & 351 & 537 & 129 & 51 & 102 & 46 & 158 & 22 & 394 & 956 & 2785 \\
\hline \multirow{5}{*}{$\mathrm{NC}$} & Shaanxi & 13 & 178 & 377 & 45 & 100 & 102 & $\begin{array}{l}40 \\
52\end{array}$ & 335 & 27 & 237 & 1523 & 2992 \\
\hline & Gansu & 31 & 70 & 221 & 76 & 20 & 122 & 20 & 231 & 9 & 228 & 440 & 1468 \\
\hline & Qinghai & 2 & 33 & 43 & 8 & 10 & 11 & 5 & 37 & 2 & 35 & 59 & 243 \\
\hline & Ningxia & 4 & 32 & 68 & 16 & 36 & 22 & 6 & 86 & 10 & 34 & 127 & 441 \\
\hline & Xinjiang & 7 & 46 & 117 & 53 & 39 & 56 & 21 & 147 & 19 & 137 & 283 & 926 \\
\hline \multicolumn{2}{|c|}{ National } & 491 & 4967 & 11172 & 2142 & 2698 & 2753 & 1131 & 9831 & 567 & 8239 & 19034 & 63024 \\
\hline
\end{tabular}


Table S12. Emissions by sectors of trace elements in China 2015 (t)

\begin{tabular}{cccccccccccc}
\hline Categories & $\mathrm{Cd}$ & $\mathrm{Co}$ & $\mathrm{Sb}$ & $\mathrm{As}$ & $\mathrm{Se}$ & $\mathrm{Ni}$ & $\mathrm{Cr}$ & $\mathrm{Cu}$ & $\mathrm{Mn}$ & $\mathrm{Pb}$ & $\mathrm{Zn}$ \\
\hline CCPP & 11 & 129 & 70 & 313 & 383 & 452 & 393 & 1147 & 2244 & 559 & 2131 \\
CCIB & 70 & 268 & 186 & 583 & 821 & 877 & 3141 & 1180 & 3117 & 3386 & 3934 \\
CCRS & 4 & 3 & 1 & 7 & 46 & 23 & 16 & 15 & 27 & 364 & 177 \\
CCOS & 22 & 155 & 94 & 492 & 295 & 158 & 663 & 774 & 1933 & 1099 & 906 \\
BBS & 31 & 2 & 19 & 27 & 28 & 220 & 224 & 54 & 137 & 478 & 203 \\
LFC & 9 & 8 & 0 & 11 & 90 & 345 & 11 & 53 & 14 & 621 & 74 \\
NFMS & 278 & 0 & 142 & 497 & 200 & 337 & 21 & 1409 & 21 & 1922 & 3933 \\
FMS & 29 & 0 & 6 & 59 & 10 & 104 & 136 & 340 & 1473 & 1081 & 5515 \\
NMMM & 29 & 0 & 2 & 147 & 821 & 174 & 214 & 287 & 620 & 1248 & 1032 \\
MSWI & 6 & 1 & 143 & 1 & 0 & 43 & 11 & 6 & 97 & 49 & 55 \\
B\&TW & 2 & 1 & 469 & 4 & 3 & 21 & 136 & 2974 & 146 & 365 & 1073 \\
Total & 491 & 567 & 1131 & 2142 & 2698 & 2753 & 4967 & 8239 & 9831 & 11172 & 19035 \\
\hline
\end{tabular}


Table S13. Spatial correlations among 11TEs and as well as with population

\begin{tabular}{|c|c|c|c|c|c|c|c|c|c|c|c|c|}
\hline & $\mathrm{Cd}$ & Co & $\mathrm{Sb}$ & As & $\mathrm{Se}$ & $\mathrm{Ni}$ & $\mathrm{Cr}$ & $\mathrm{Cu}$ & $\mathrm{Mn}$ & $\mathrm{Pb}$ & $\mathrm{Zn}$ & Population \\
\hline $\mathrm{Cd}$ & 1 & $.999^{* *}$ & $.955^{* *}$ & $.999^{* *}$ & $1.000^{* *}$ & $1.000^{* *}$ & $1.000^{* *}$ & $.985^{* *}$ & $1.000^{* *}$ & $1.000^{* *}$ & $1.000^{* *}$ & $.787^{* *}$ \\
\hline $\mathrm{Co}$ & & 1 & $.950^{* *}$ & $1.000^{* *}$ & $1.000^{* *}$ & $.999^{* *}$ & $1.000^{* *}$ & $.982^{* *}$ & $1.000^{* *}$ & $.999^{* *}$ & $1.000^{* *}$ & $.775^{* *}$ \\
\hline $\mathrm{Sb}$ & & & 1 & $.950^{* *}$ & $.951^{* *}$ & $.957^{* *}$ & $.956^{* *}$ & $.992^{* *}$ & $.953^{* *}$ & $.961^{* *}$ & $.959^{* *}$ & $.934^{* *}$ \\
\hline As & & & & 1 & $1.000^{* *}$ & $.999^{* *}$ & $1.000^{* *}$ & $.982^{* *}$ & $1.000^{* *}$ & $.999^{* *}$ & $1.000^{* *}$ & $.775^{* *}$ \\
\hline $\mathrm{Se}$ & & & & & 1 & $.999^{* *}$ & $1.000^{* *}$ & $.983^{* *}$ & $1.000^{* *}$ & $.999^{* *}$ & $1.000^{* *}$ & $.779^{* *}$ \\
\hline $\mathrm{Hg}$ & & & & & & $.999^{* *}$ & $.999^{* *}$ & $.991^{* *}$ & $.999^{* *}$ & $1.000^{* *}$ & $1.000^{* *}$ & $.810^{* *}$ \\
\hline $\mathrm{Ni}$ & & & & & & 1 & $1.000^{* *}$ & $.986^{* *}$ & $.999^{* *}$ & $1.000^{* *}$ & $.999^{* *}$ & $.791^{* *}$ \\
\hline $\mathrm{Cr}$ & & & & & & & 1 & $.985^{* *}$ & $1.000^{* *}$ & $1.000^{* *}$ & $1.000^{* *}$ & $.787^{* *}$ \\
\hline $\mathrm{Cu}$ & & & & & & & & 1 & $.984^{* *}$ & $.988^{* *}$ & $.987^{* *}$ & $.882^{* *}$ \\
\hline $\mathrm{Mn}$ & & & & & & & & & 1 & $1.000^{* *}$ & $1.000^{* *}$ & $.782^{* *}$ \\
\hline $\mathrm{Pb}$ & & & & & & & & & & 1 & $1.000^{* *}$ & $.798^{* *}$ \\
\hline $\mathrm{Zn}$ & & & & & & & & & & & 1 & $.793^{* *}$ \\
\hline Population & & & & & & & & & & & . & 1 \\
\hline
\end{tabular}

** is significantly correlated at the.01 level (bilateral) 
Table S14. The population-weighted concentrations (PWC) and the maximum gridded concentrations (MGC) of 11 TEs in different regions of China in 2012 and 2015

\begin{tabular}{|c|c|c|c|c|c|c|c|c|}
\hline Species & Year $\left(\mathrm{ng} / \mathrm{m}^{3}\right)$ & National & $\mathrm{NC}$ & NEC & $\mathrm{EC}$ & $\mathrm{CSC}$ & SWC & NWC \\
\hline \multirow{5}{*}{$\mathrm{Cd}$} & PWC-2012 & 0.86 & 0.92 & 0.75 & 1.07 & 0.81 & 0.71 & 0.50 \\
\hline & PWC-2015 & 0.77 & 0.72 & 0.71 & 1.04 & 0.73 & 0.68 & 0.46 \\
\hline & Reduction & $9.8 \%$ & $22.3 \%$ & $6.3 \%$ & $2.5 \%$ & $10.0 \%$ & $3.8 \%$ & $6.9 \%$ \\
\hline & MGC-2012 & 4.01 & 2.26 & 2.01 & 2.85 & 2.48 & 4.01 & 2.76 \\
\hline & MGC-2015 & 2.69 & 1.89 & 1.64 & 2.60 & 1.89 & 2.69 & 1.99 \\
\hline \multirow{5}{*}{$\mathrm{Co}$} & PWC-2012 & 0.68 & 0.97 & 0.62 & 0.82 & 0.56 & 0.47 & 0.63 \\
\hline & PWC-2015 & 0.48 & 0.51 & 0.37 & 0.50 & 0.32 & 0.37 & 0.32 \\
\hline & Reduction & $29.2 \%$ & $47.6 \%$ & $39.4 \%$ & $38.5 \%$ & $43.8 \%$ & $22.8 \%$ & $48.6 \%$ \\
\hline & MGC-2012 & 4.81 & 2.89 & 2.26 & 2.79 & 1.76 & 3.30 & 4.81 \\
\hline & MGC-2015 & 2.18 & 1.32 & 1.50 & 1.97 & 0.14 & 2.54 & 2.18 \\
\hline \multirow{5}{*}{$\mathrm{Sb}$} & PWC-2012 & 1.78 & 2.58 & 1.24 & 2.20 & 1.68 & 1.12 & 1.28 \\
\hline & PWC-2015 & 1.62 & 2.15 & 1.10 & 2.11 & 1.49 & 1.08 & 1.17 \\
\hline & Reduction & $9.2 \%$ & $16.8 \%$ & $11.5 \%$ & $4.4 \%$ & $11.1 \%$ & $4.1 \%$ & $8.0 \%$ \\
\hline & MGC-2012 & 11.80 & 11.80 & 3.11 & 7.86 & 4.15 & 3.82 & 6.43 \\
\hline & MGC-2015 & 10.30 & 10.30 & 2.71 & 6.48 & 3.82 & 3.36 & 5.37 \\
\hline \multirow{5}{*}{ As } & PWC-2012 & 3.59 & 4.64 & 3.61 & 4.68 & 3.24 & 2.17 & 1.93 \\
\hline & PWC-2015 & 2.94 & 3.78 & 3.24 & 4.09 & 2.50 & 1.94 & 1.59 \\
\hline & Reduction & $18.1 \%$ & $18.6 \%$ & $10.4 \%$ & $12.6 \%$ & $22.7 \%$ & $10.8 \%$ & $17.6 \%$ \\
\hline & MGC-2012 & 15.88 & 14.38 & 12.63 & 15.88 & 9.95 & 9.89 & 8.85 \\
\hline & MGC-2015 & 13.88 & 12.88 & 10.14 & 13.88 & 7.09 & 6.81 & 5.08 \\
\hline \multirow{5}{*}{$\mathrm{Se}$} & PWC-2012 & 6.56 & 10.24 & 5.00 & 7.89 & 6.33 & 4.04 & 3.60 \\
\hline & PWC-2015 & 5.78 & 8.67 & 4.75 & 7.46 & 5.10 & 3.92 & 3.30 \\
\hline & Reduction & $11.9 \%$ & $15.3 \%$ & $5.1 \%$ & $5.5 \%$ & $19.5 \%$ & $2.9 \%$ & $8.1 \%$ \\
\hline & MGC-2012 & 27.30 & 27.30 & 13.60 & 17.70 & 19.30 & 18.40 & 18.50 \\
\hline & MGC-2015 & 21.60 & 21.60 & 12.10 & 16.30 & 12.90 & 13.60 & 15.60 \\
\hline \multirow{5}{*}{$\mathrm{Ni}$} & PWC-2012 & 4.06 & 4.68 & 3.58 & 5.52 & 3.67 & 2.52 & 2.48 \\
\hline & PWC-2015 & 3.55 & 3.79 & 3.12 & 5.14 & 3.03 & 2.48 & 2.14 \\
\hline & Reduction & $12.5 \%$ & $18.9 \%$ & $13.0 \%$ & $6.9 \%$ & $17.4 \%$ & $1.6 \%$ & $13.6 \%$ \\
\hline & MGC-2012 & 32.88 & 12.14 & 11.39 & 32.88 & 10.23 & 9.21 & 12.20 \\
\hline & MGC-2015 & 29.7 & 12.8 & 9.0 & 29.7 & 7.33 & 6.84 & 9.33 \\
\hline \multirow{5}{*}{$\mathrm{Cr}$} & PWC-2012 & 16.28 & 21.94 & 14.64 & 19.92 & 14.44 & 11.90 & 11.07 \\
\hline & PWC-2015 & 10.49 & 12.84 & 9.81 & 13.84 & 8.72 & 9.00 & 6.83 \\
\hline & Reduction & $35.6 \%$ & $41.5 \%$ & $33.0 \%$ & $30.5 \%$ & $39.6 \%$ & $24.4 \%$ & $38.2 \%$ \\
\hline & MGC-2012 & 60.8 & 55.8 & 42.4 & 51.5 & 39.8 & 56.1 & 60.8 \\
\hline & MGC-2015 & 33.5 & 31.5 & 26.3 & 33.5 & 19.6 & 34.1 & 28.6 \\
\hline \multirow{5}{*}{$\mathrm{Cu}$} & PWC-2012 & 17.54 & 27.16 & 12.95 & 21.83 & 16.36 & 10.39 & 10.37 \\
\hline & PWC-2015 & 15.80 & 23.65 & 12.35 & 20.56 & 14.79 & 10.14 & 9.21 \\
\hline & Reduction & $9.9 \%$ & $12.9 \%$ & $4.7 \%$ & $5.8 \%$ & $9.6 \%$ & $2.4 \%$ & $11.1 \%$ \\
\hline & MGC-2012 & 79.90 & 79.90 & 32.80 & 74.00 & 41.80 & 41.10 & 48.50 \\
\hline & MGC-2015 & 74.00 & 74.00 & 25.20 & 60.20 & 35.30 & 32.50 & 32.20 \\
\hline \multirow{5}{*}{$\mathrm{Mn}$} & PWC-2012 & 26.40 & 40.45 & 22.98 & 33.37 & 23.06 & 12.71 & 22.12 \\
\hline & PWC-2015 & 19.21 & 24.07 & 19.03 & 26.91 & 16.52 & 10.80 & 13.71 \\
\hline & Reduction & $27.2 \%$ & $40.5 \%$ & $17.2 \%$ & $19.4 \%$ & $28.4 \%$ & $15.0 \%$ & $38.0 \%$ \\
\hline & MGC-2012 & 107.5 & 107.5 & 65.70 & 85.70 & 62.40 & 50.50 & 132.8 \\
\hline & MGC-2015 & 68.30 & 58.60 & 50.10 & 68.30 & 35.10 & 31.20 & 56.10 \\
\hline \multirow{5}{*}{$\mathrm{Pb}$} & PWC-2012 & 27.99 & 44.18 & 22.97 & 34.26 & 24.87 & 17.46 & 17.75 \\
\hline & PWC-2015 & 21.59 & 29.57 & 18.27 & 28.33 & 19.00 & 15.71 & 12.43 \\
\hline & Reduction & $22.9 \%$ & $33.1 \%$ & $20.5 \%$ & $17.3 \%$ & $23.6 \%$ & $10.0 \%$ & $30.0 \%$ \\
\hline & MGC-2012 & 142.6 & 142.6 & 73.88 & 104.8 & 79.75 & 70.90 & 91.90 \\
\hline & MGC-2015 & 88.63 & 88.63 & 52.75 & 78.63 & 55.63 & 48.30 & 53.80 \\
\hline \multirow{5}{*}{$\mathrm{Zn}$} & PWC-2012 & 28.98 & 50.61 & 21.22 & 35.80 & 24.81 & 12.69 & 27.24 \\
\hline & PWC-2015 & 25.13 & 37.77 & 19.03 & 32.61 & 21.50 & 11.53 & 25.89 \\
\hline & Reduction & $13.3 \%$ & $25.4 \%$ & $10.3 \%$ & $8.9 \%$ & $13.3 \%$ & $9.2 \%$ & $5.0 \%$ \\
\hline & MGC-2012 & 160.8 & 124.1 & 47.3 & 88.3 & 67.3 & 36.8 & 160.9 \\
\hline & MGC-2015 & 157.7 & 83.9 & 39.3 & 74.3 & 46.4 & 27.5 & 157.8 \\
\hline
\end{tabular}



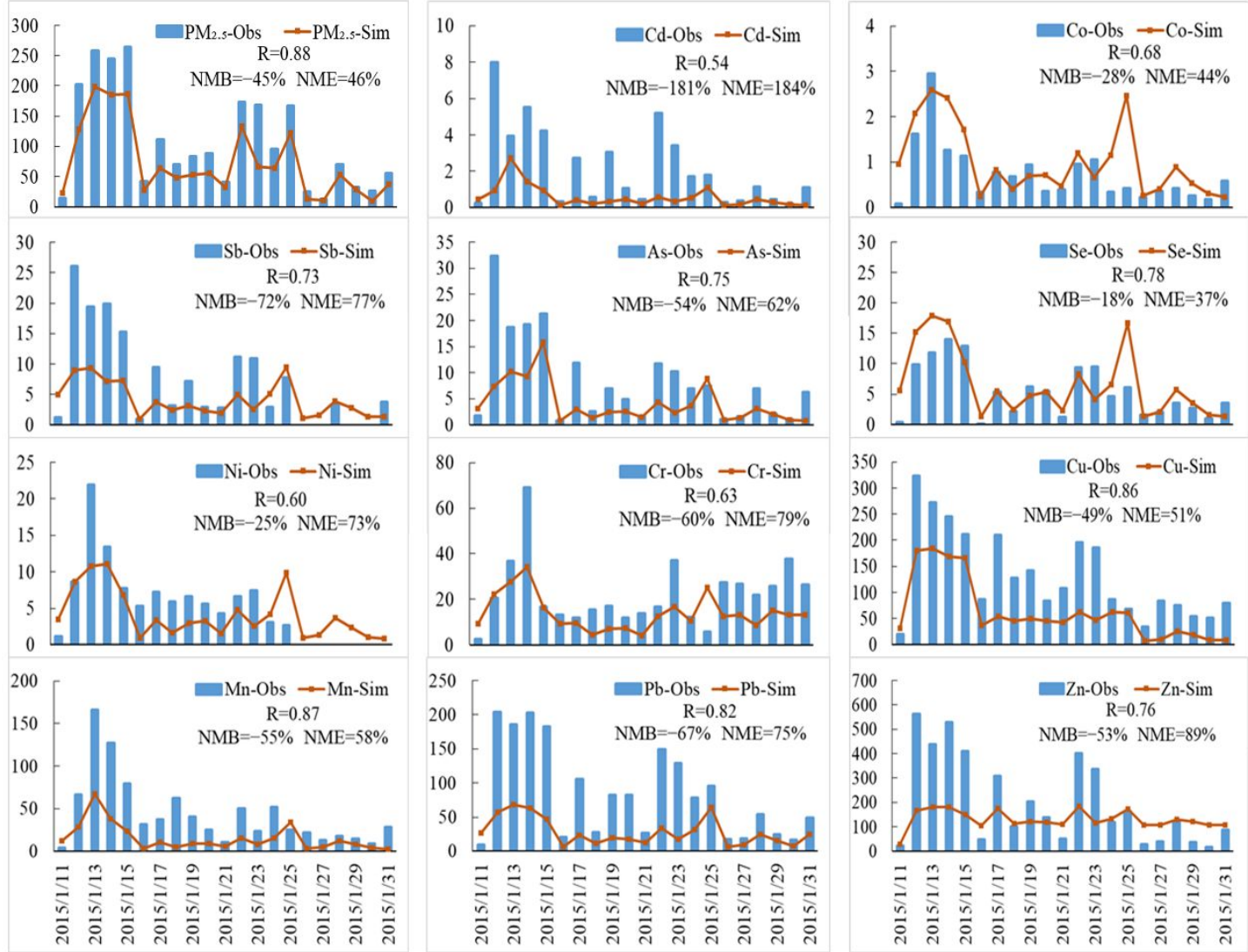

Figure S1. Time series of observed and predicted daily concentrations for $\mathrm{PM}_{2.5}\left(\mu \mathrm{g} / \mathrm{m}^{3}\right)$ and trace elements $\left(\mathrm{ng} / \mathrm{m}^{3}\right)$ in BNU site in January, 2015. R, NMB, and NME represents the correlation coefficient, normalized mean bias, and normalized mean error, respectively. 


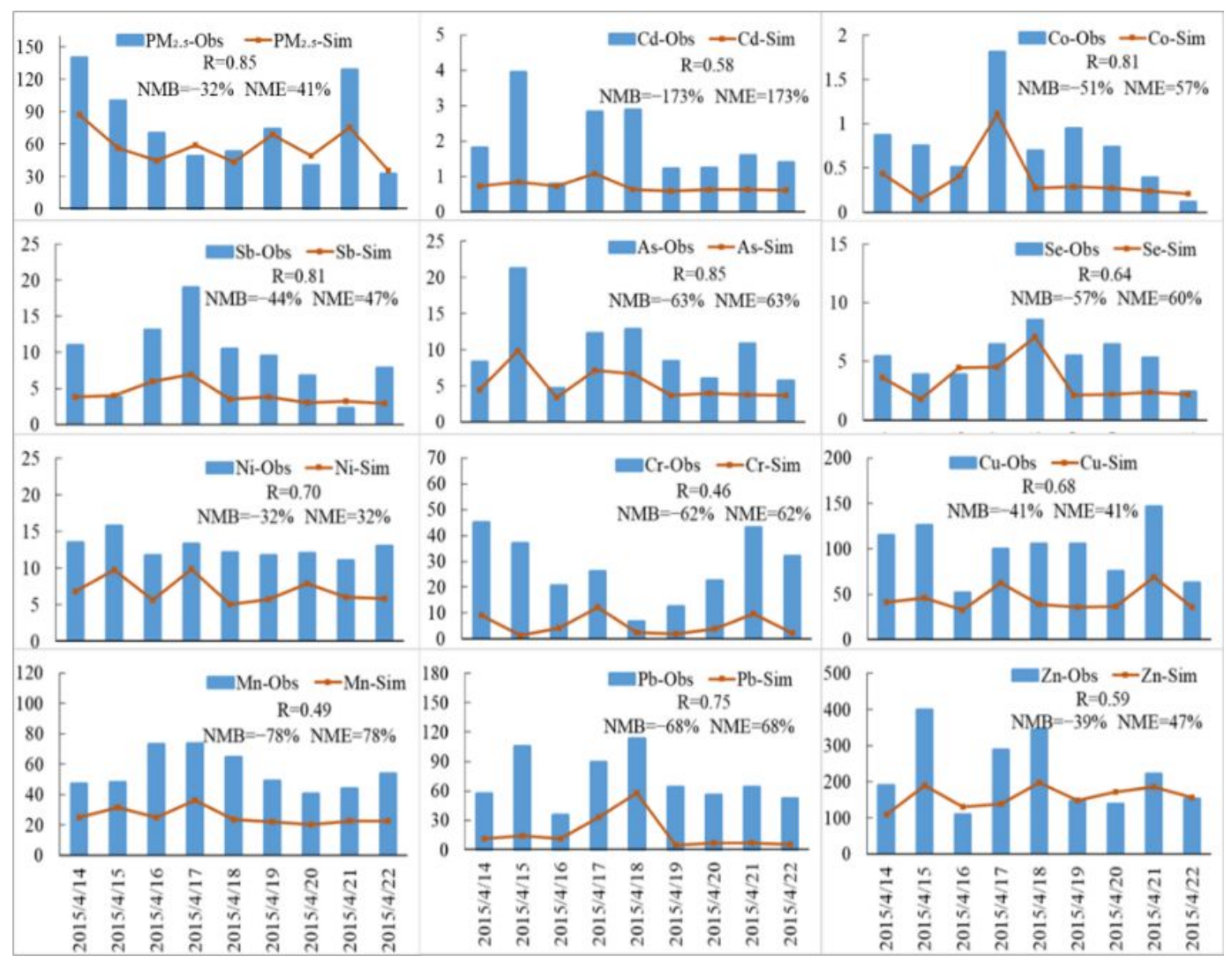

Figure S2. Time series of observed and predicted daily concentrations for $\mathrm{PM}_{2.5}\left(\mu \mathrm{g} / \mathrm{m}^{3}\right)$ and trace elements $\left(\mathrm{ng} / \mathrm{m}^{3}\right)$ in BNU site in April, 2015. R, NMB, and NME represents the correlation coefficient, normalized mean bias, and normalized mean error, respectively. 


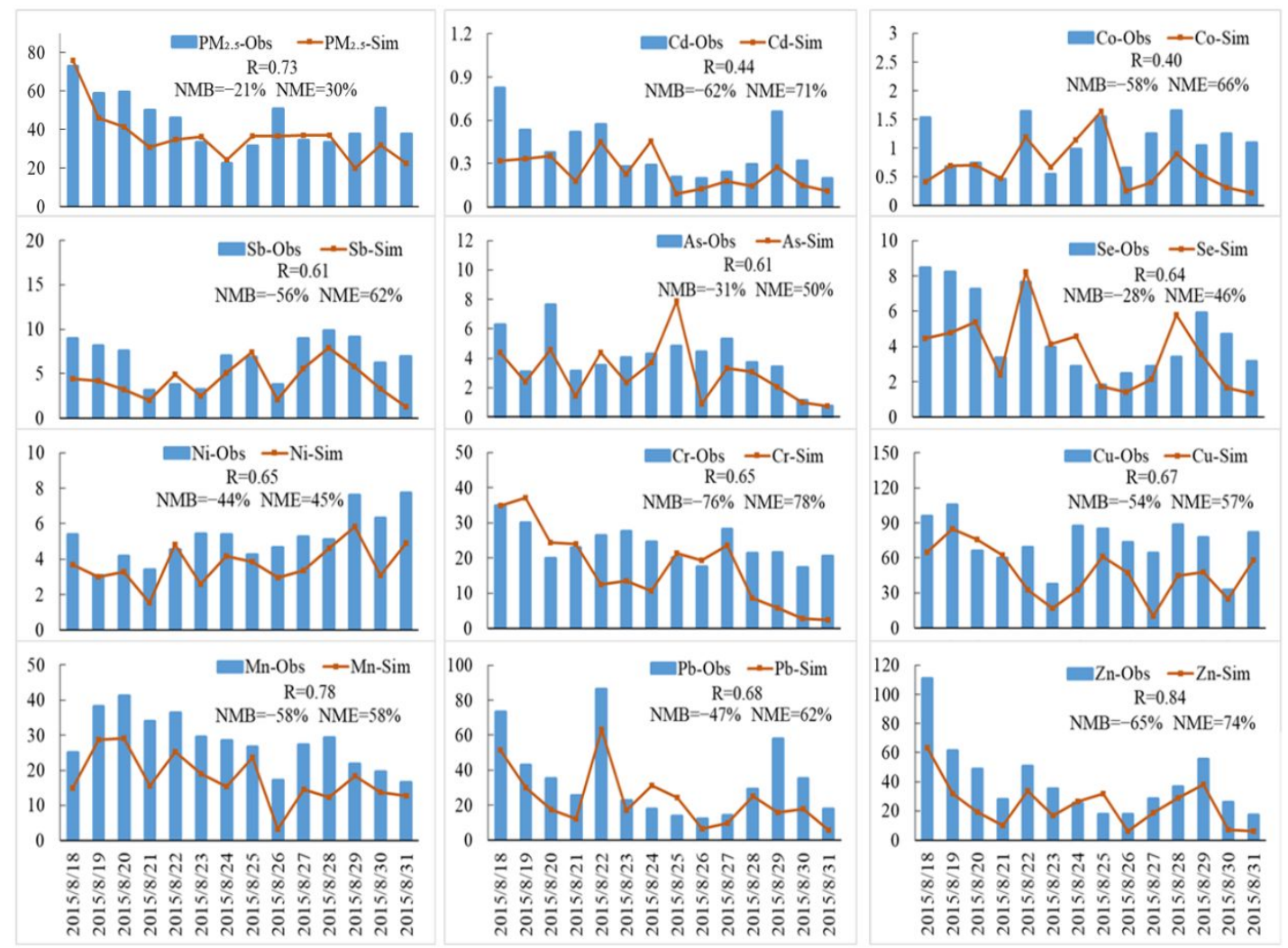

Figure S3. Time series of observed and predicted daily concentrations for $\mathrm{PM}_{2.5}\left(\mu \mathrm{g} / \mathrm{m}^{3}\right)$ and trace elements $\left(\mathrm{ng} / \mathrm{m}^{3}\right)$ in BNU site in August, 2015. R, NMB, and NME represents the correlation coefficient, normalized mean bias, and normalized mean error, respectively. 


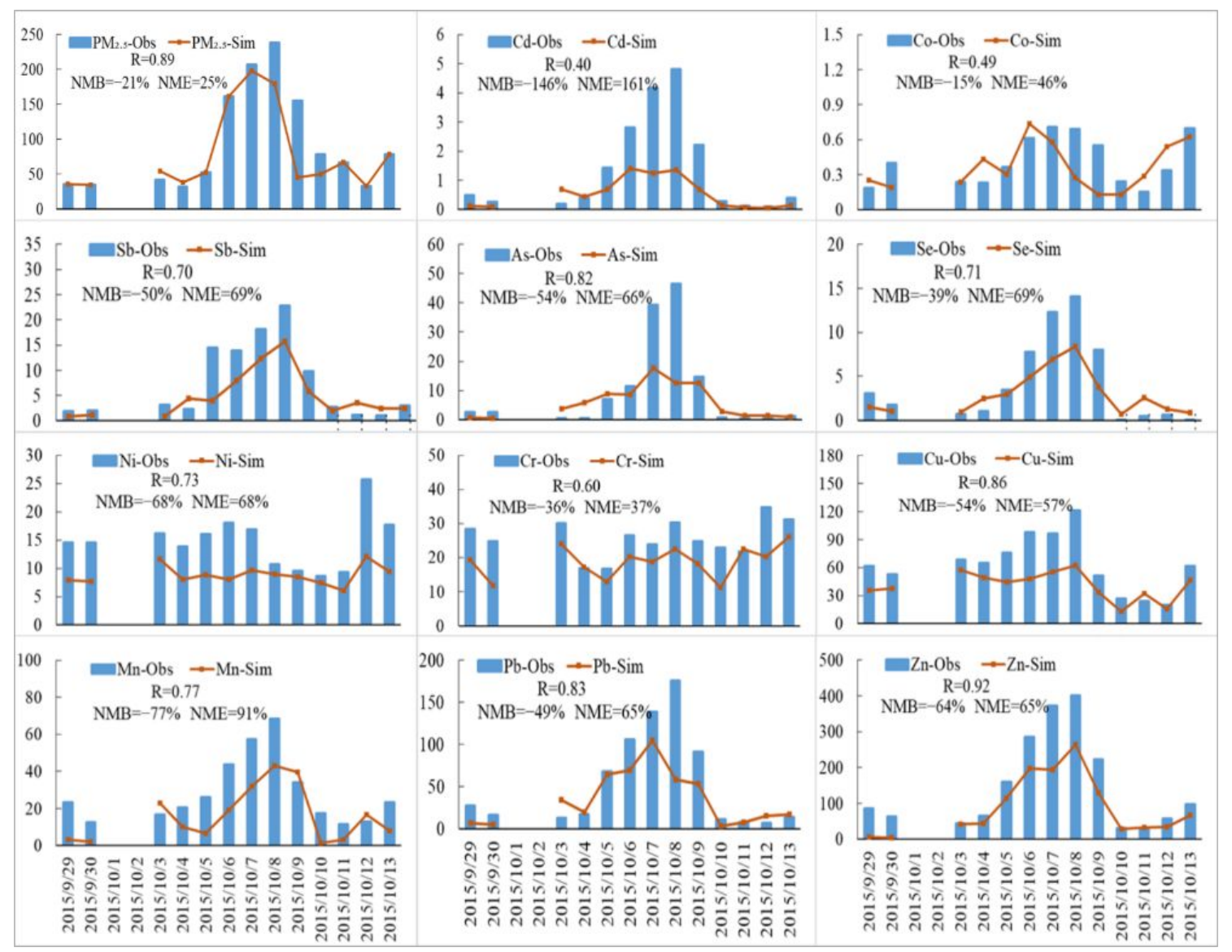

Figure S4. Time series of observed and predicted daily concentrations for $\mathrm{PM}_{2.5}\left(\mu \mathrm{g} / \mathrm{m}^{3}\right)$ and trace elements $\left(\mathrm{ng} / \mathrm{m}^{3}\right)$ in BNU site in October, 2015. R, NMB, and NME represents the correlation coefficient, normalized mean bias, and normalized mean error, respectively. 
a

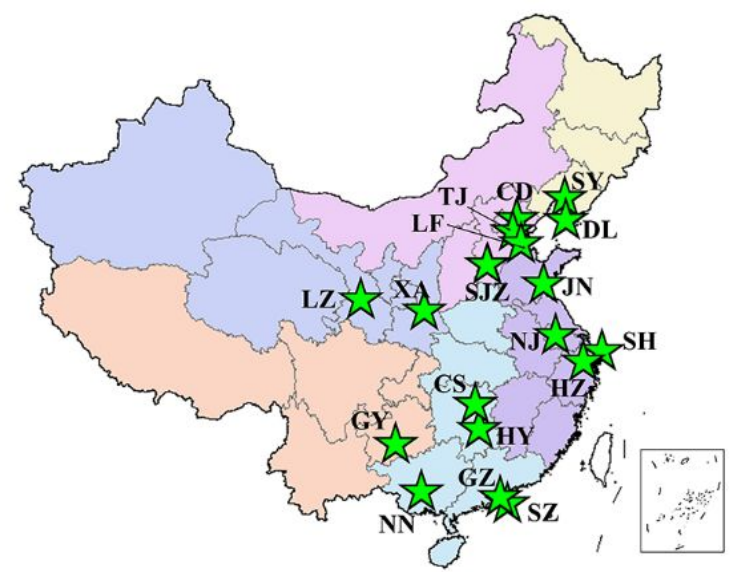

b

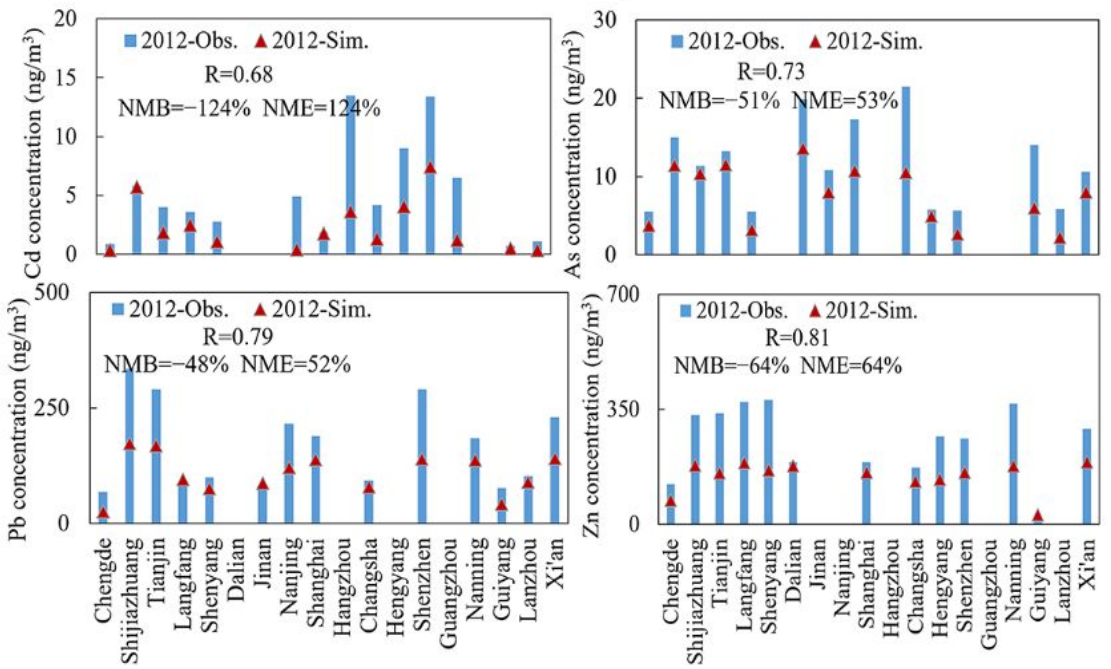

Figure S5. Comparison between simulated and observed TEs concentrations in multiple cities for 2012. (a) The geographic location of multiple cities in China. Chengde (CD), Shijiazhuang (SJZ), Tianjin (TJ), Langfang (LF), Shenyang (SY), Dalian (DL), Nanjing (NJ), Shanghai (SH), Hangzhou (HZ), Jinan (JN), Hengyang (HY), Changsha (CS), Shenzhen (SZ), Guangzhou (GZ), Nanning (NN), Guiyang (GY), Lanzhou (LZ), Xi'an (XA). (b) The simulated and observed mean concentrations of TEs in each city. $\mathrm{R}, \mathrm{NMB}$, and NME represents the correlation coefficient, normalized mean bias, and normalized mean error, respectively. 
a
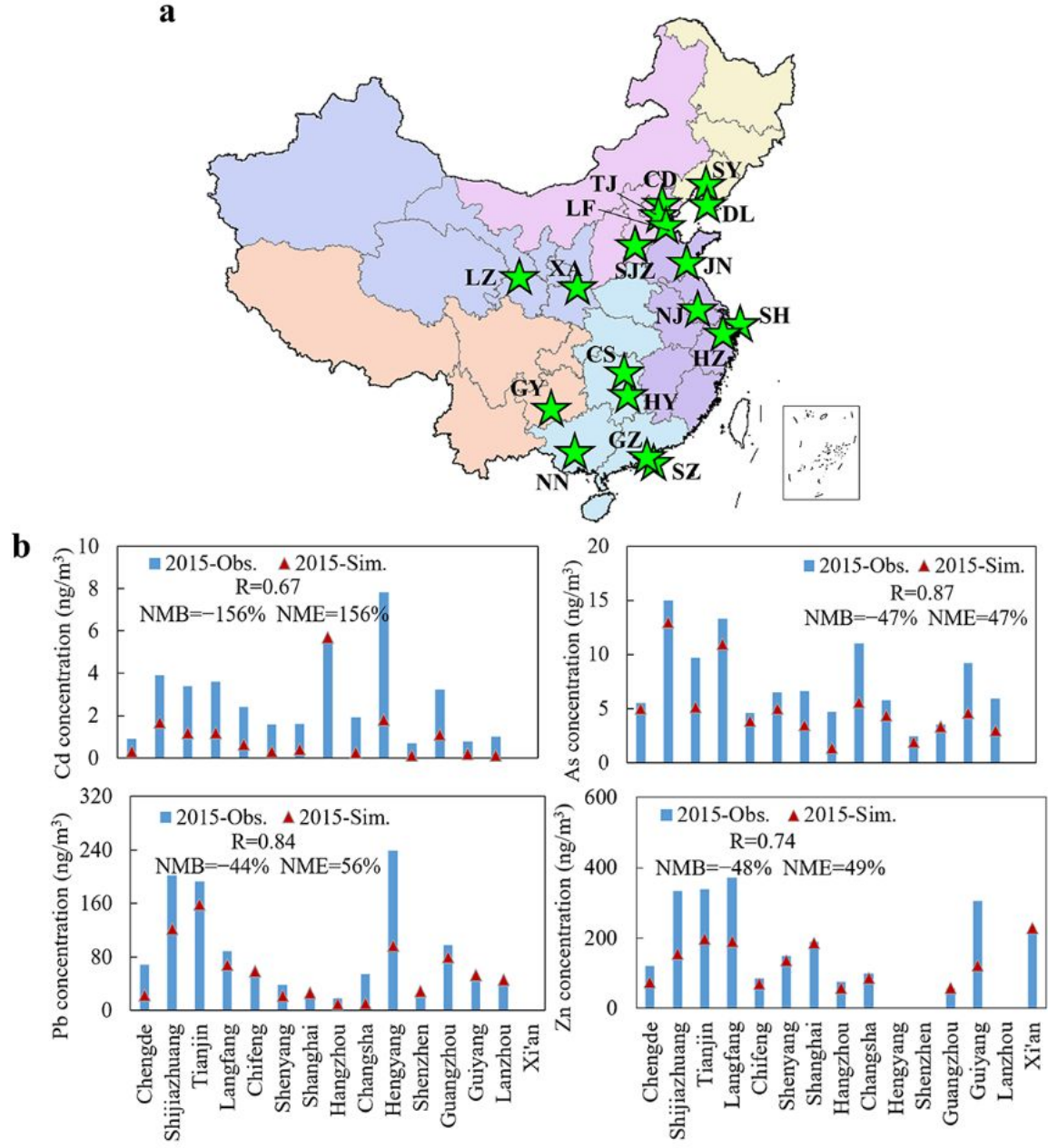

Figure S6. Comparison between simulated and observed TEs concentrations in multiple cities for 2015. (a) The geographic location of multiple cities in China. Chengde (CD), Shijiazhuang (SJZ), Tianjin (TJ), Langfang (LF), Shenyang (SY), Dalian (DL), Nanjing (NJ), Shanghai (SH), Hangzhou (HZ), Jinan (JN), Hengyang (HY), Changsha (CS), Shenzhen (SZ), Guangzhou (GZ), Nanning (NN), Guiyang (GY), Lanzhou (LZ), Xi'an (XA). (b) The simulated and observed mean concentrations of TEs in each city. $\mathrm{R}, \mathrm{NMB}$, and NME represents the correlation coefficient, normalized mean bias, and normalized mean error, respectively. 

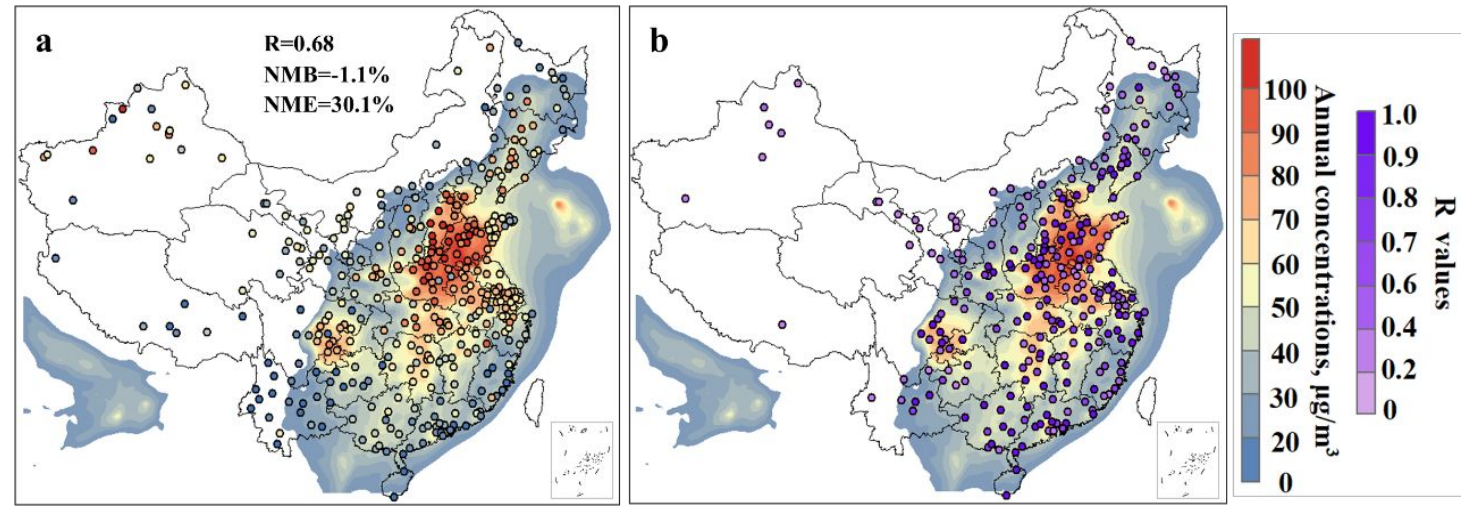

Figure S7. Comparison of spatial variations between simulated and surface observed $\mathrm{PM}_{2.5}$ concentrations in 2015 in mainland China. (a) Spatial variations of simulated annual mean $\mathrm{PM}_{2.5}$ concentrations in 2015. Monitored annual mean $\mathrm{PM}_{2.5}$ concentrations in 366 cities are overlaid. R, $\mathrm{NMB}$, and NME represents the correlation coefficient, normalized mean bias, and normalized mean error, respectively. (b) Spatial variations of simulated annual mean $\mathrm{PM}_{2.5}$ concentrations in 2015 . The correlation coefficients between simulated and observed daily $\mathrm{PM}_{2.5}$ concentration in 2015 are overlaid for each city. 


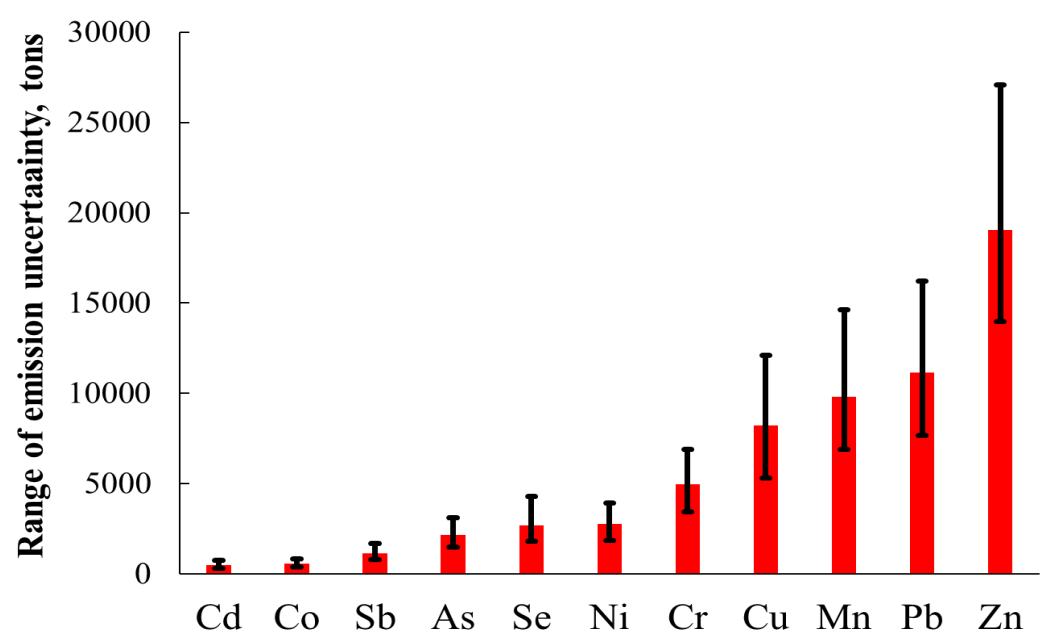

Figure S8. Uncertainty analysis of TEs emissions in China, 2015. 


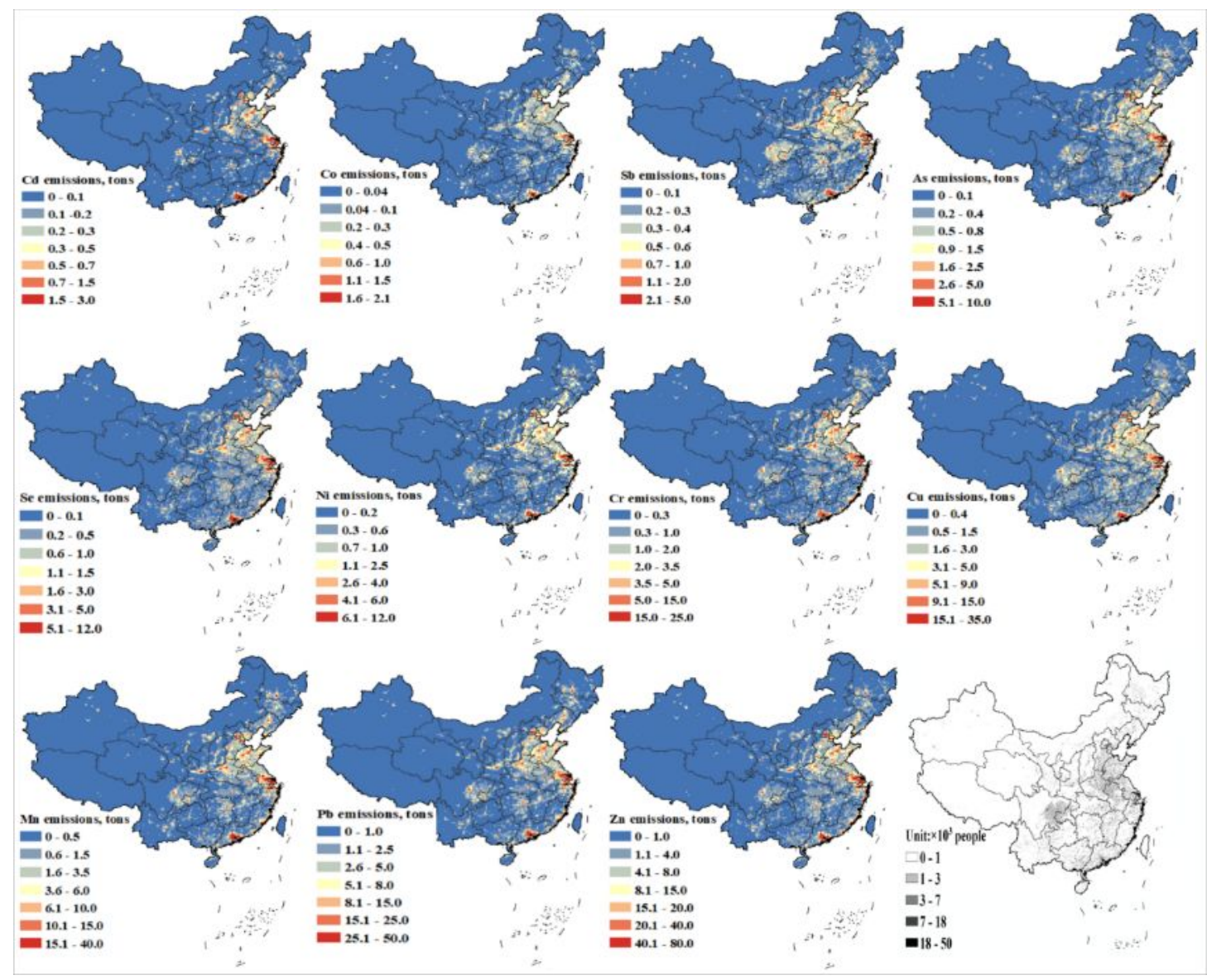

Figure S9. Spatial distribution characteristics of TEs emissions in 2015 


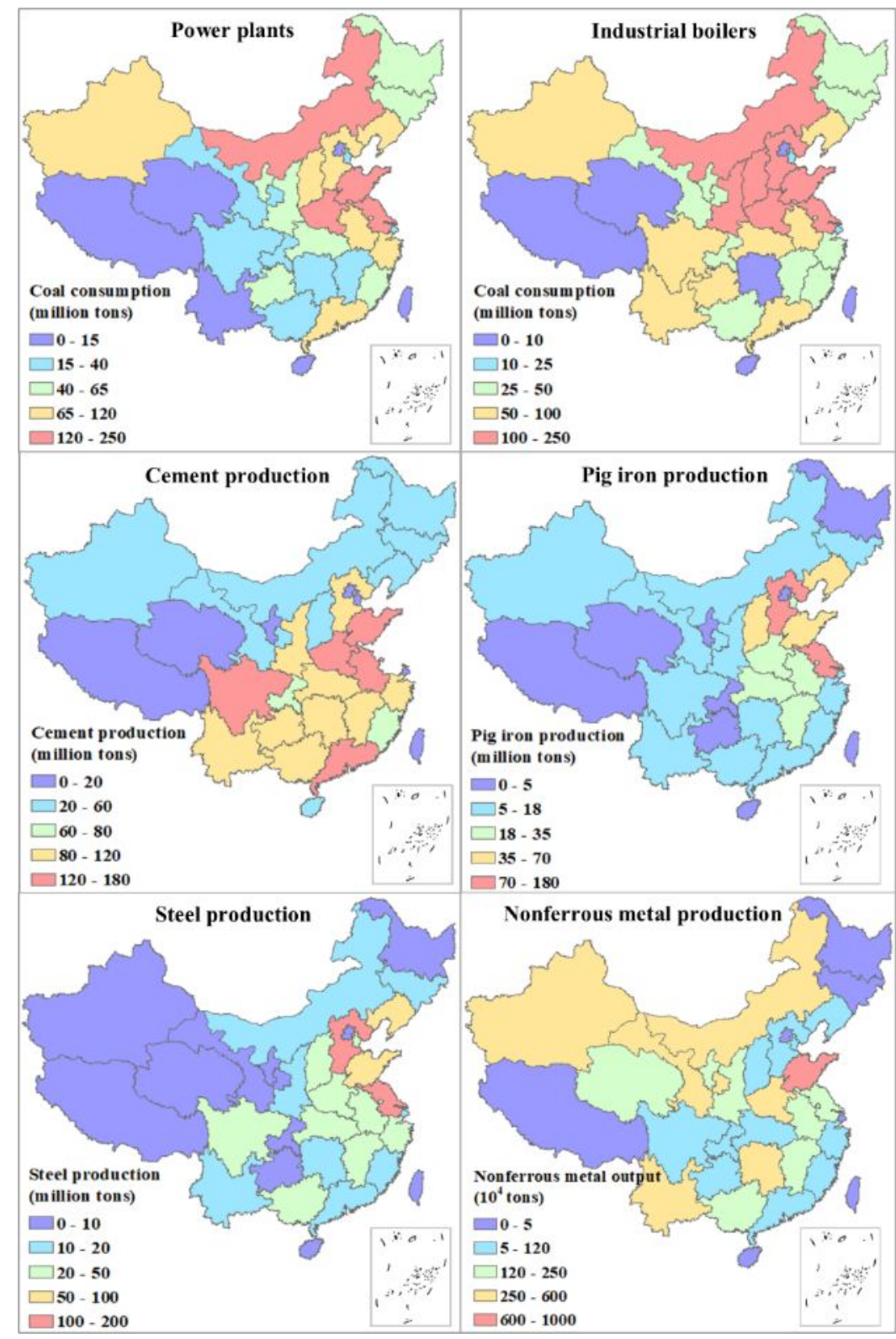

Figure S10. Spatial distribution of activity level of important industries in China in 2015. 

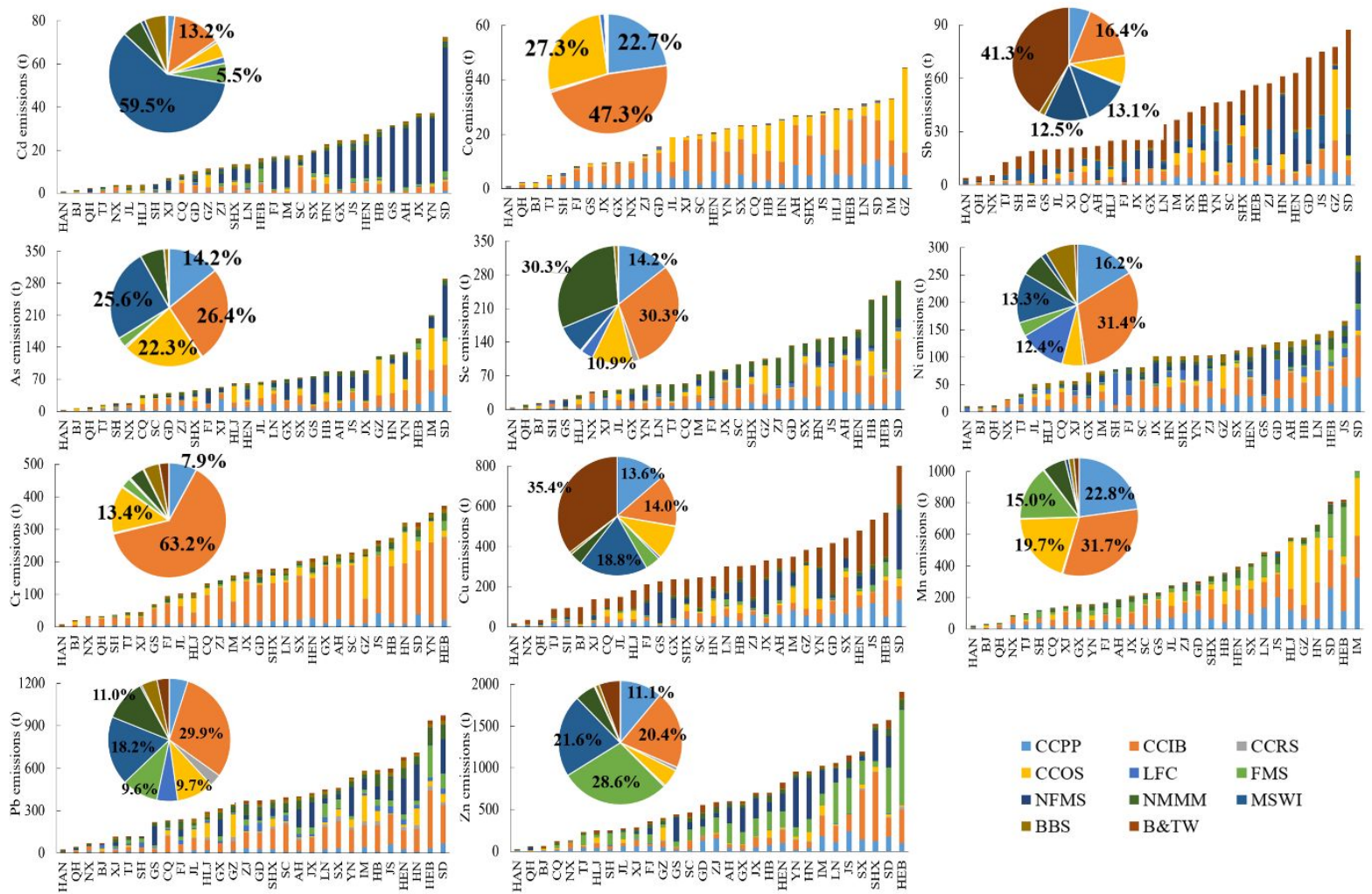

Figure S11. Provincial TEs emissions from anthropogenic sources and national composition by source categories in 2015 

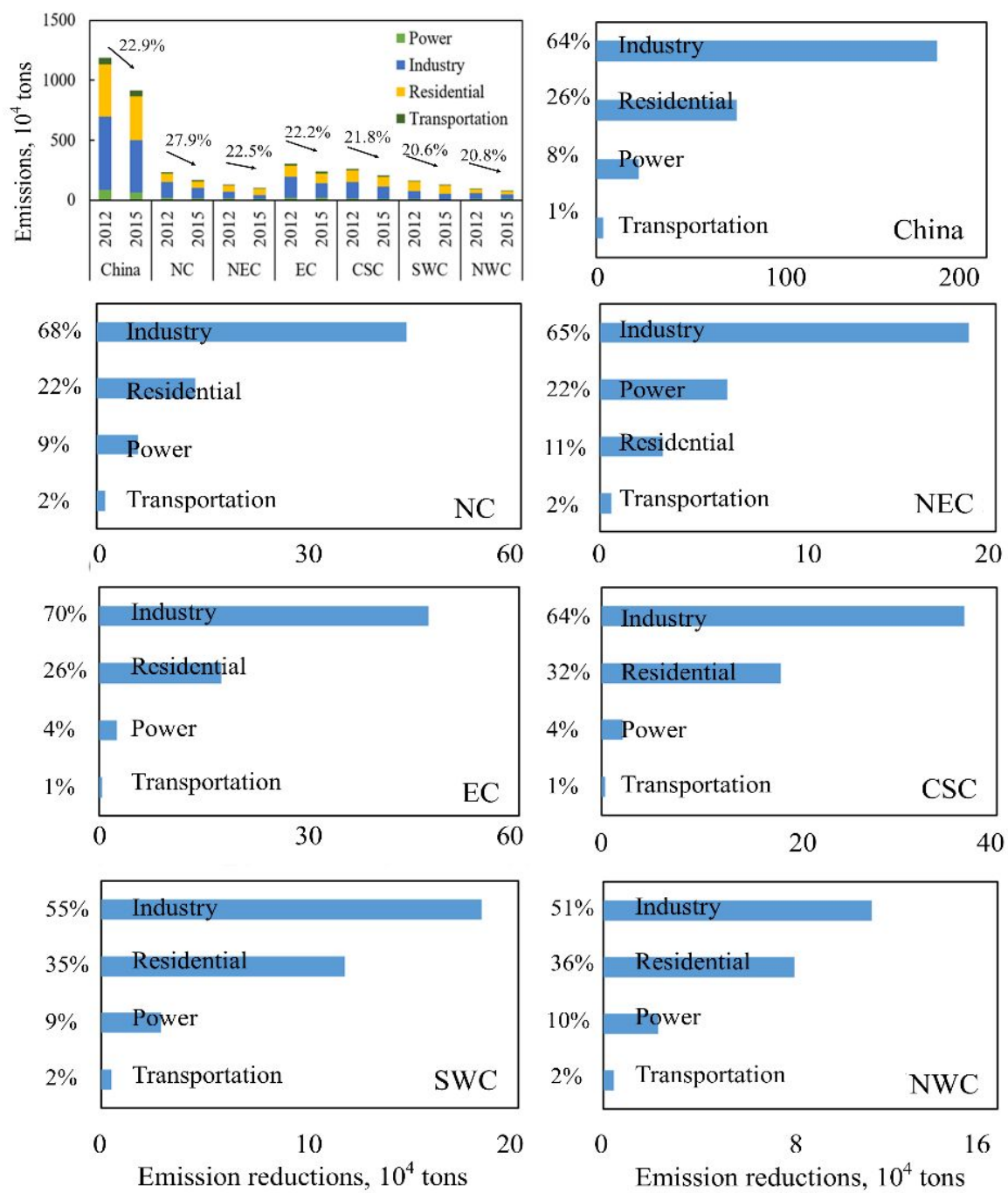

Figure $\mathrm{S} 12 . \mathrm{PM}_{2.5}$ emission changes in China and the six regions of China 
a

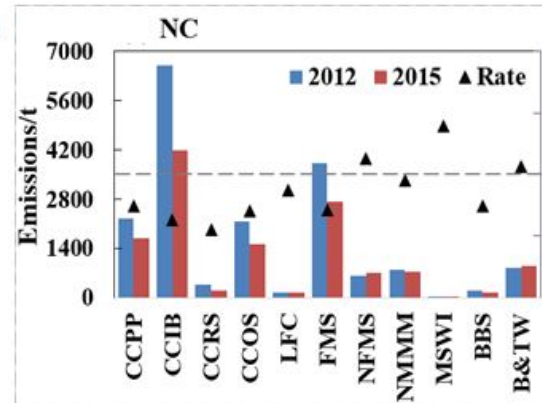

c

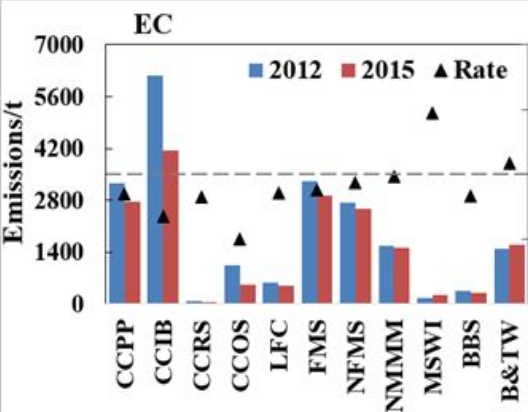

e

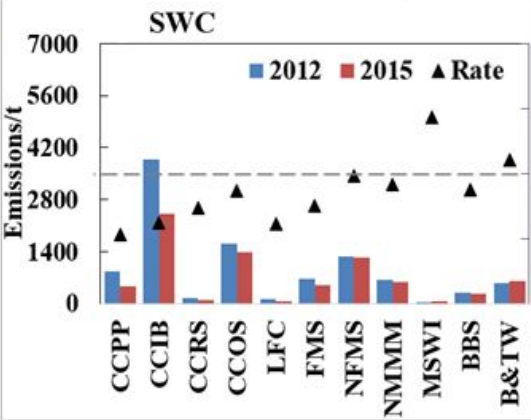

b

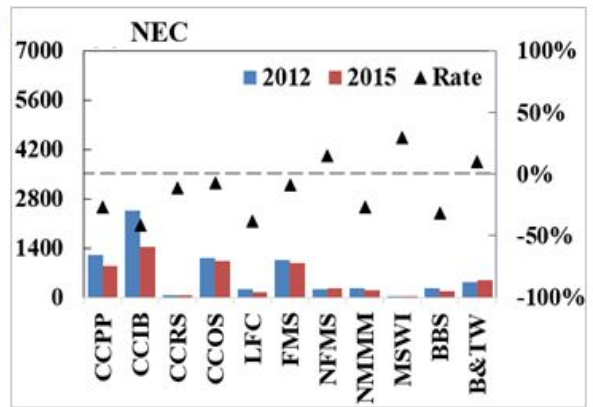

d
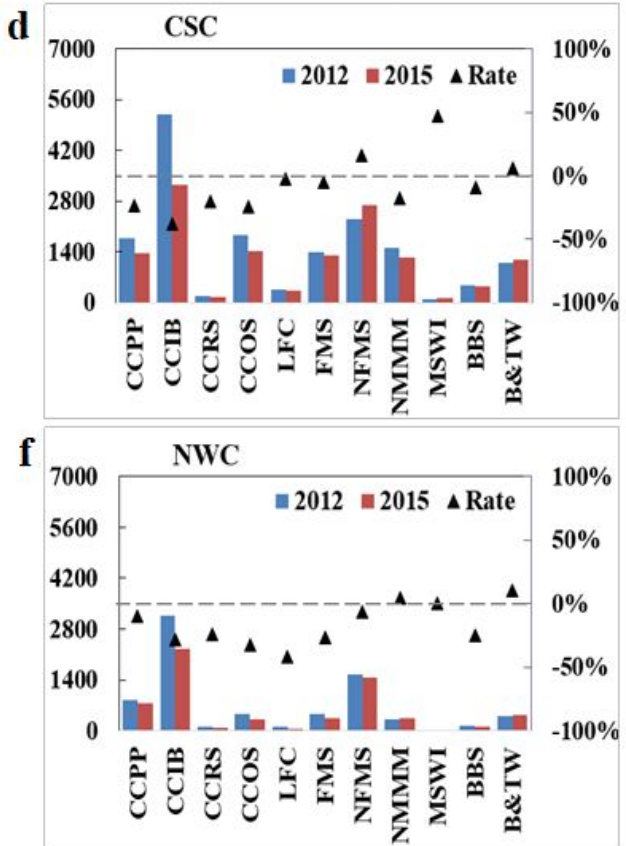

Figure S13. Comparisons of the total TEs emissions in China in the years of 2012 and 2015 by region. We defined Rate in $\mathrm{a}-\mathrm{f}$ as the differences between 2015 and 2012 emissions (2015-2012) divided by 2012 emissions; Total 11 TEs emissions by sectors in 2012 and 2015 by regions 

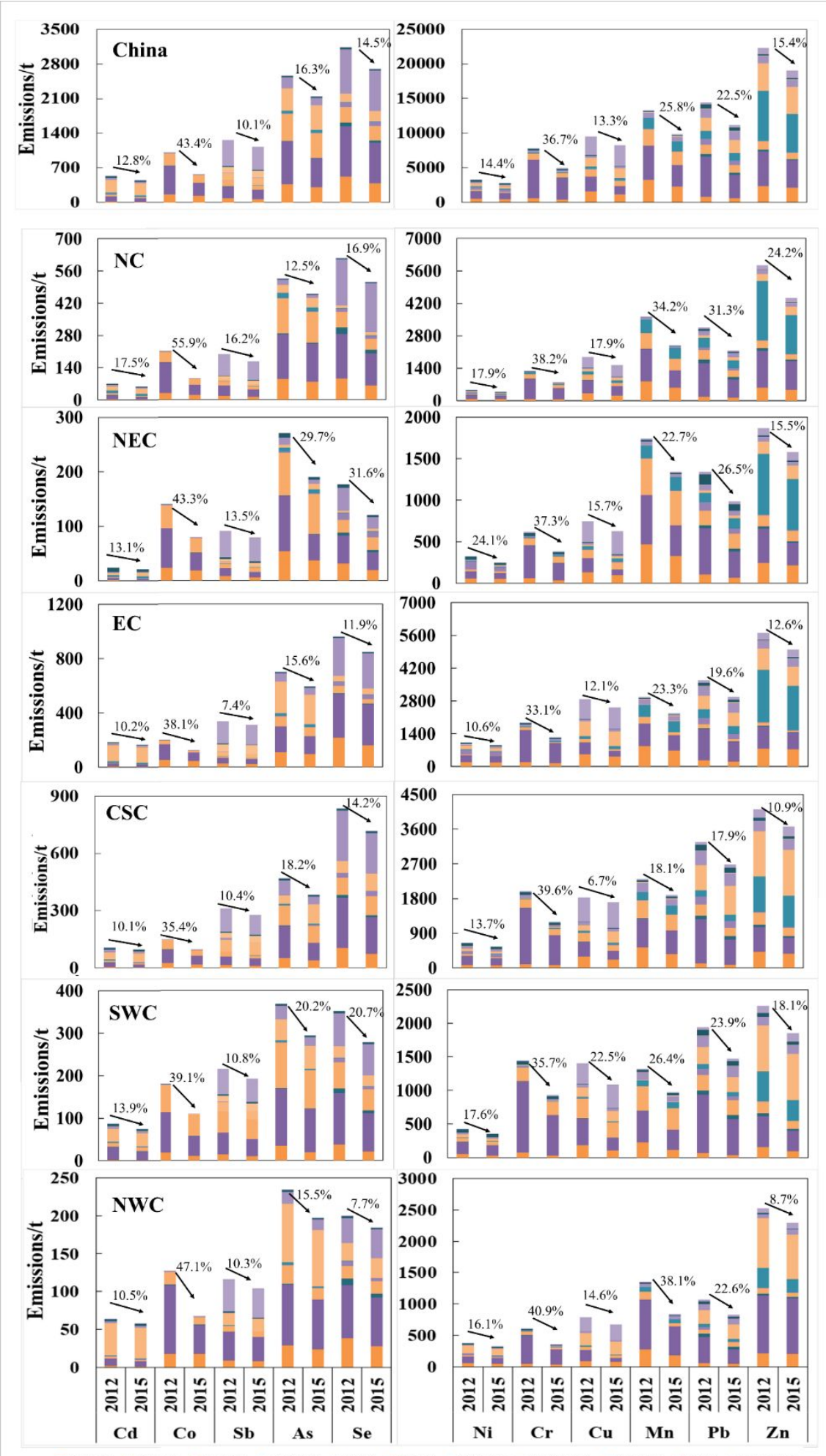

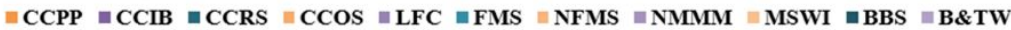

Figure S14. Comparisons of each trace element emissions by sectors in 2012 and 2015 . We defined the percentage values above the bar chart in (a) - (g) as 2015 emission minus 2012 emission (2015-2012) divided by 2012 emission for each trace element 


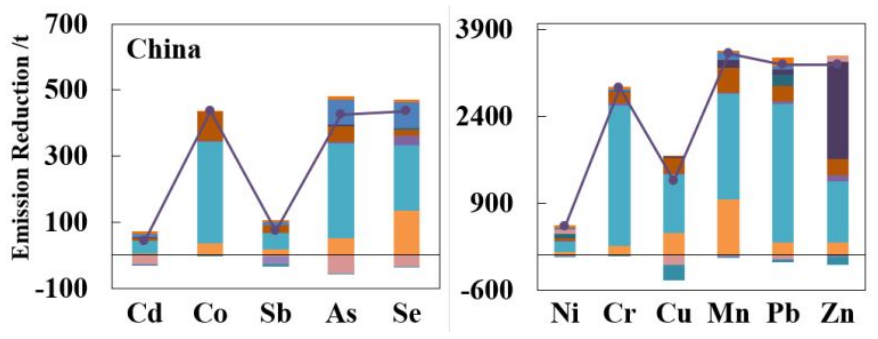

Figure S15. Emission reductions by sectors of each trace element from 2012-2015 in China. Purple dots on lines in each figure represent total reduction of each trace element 


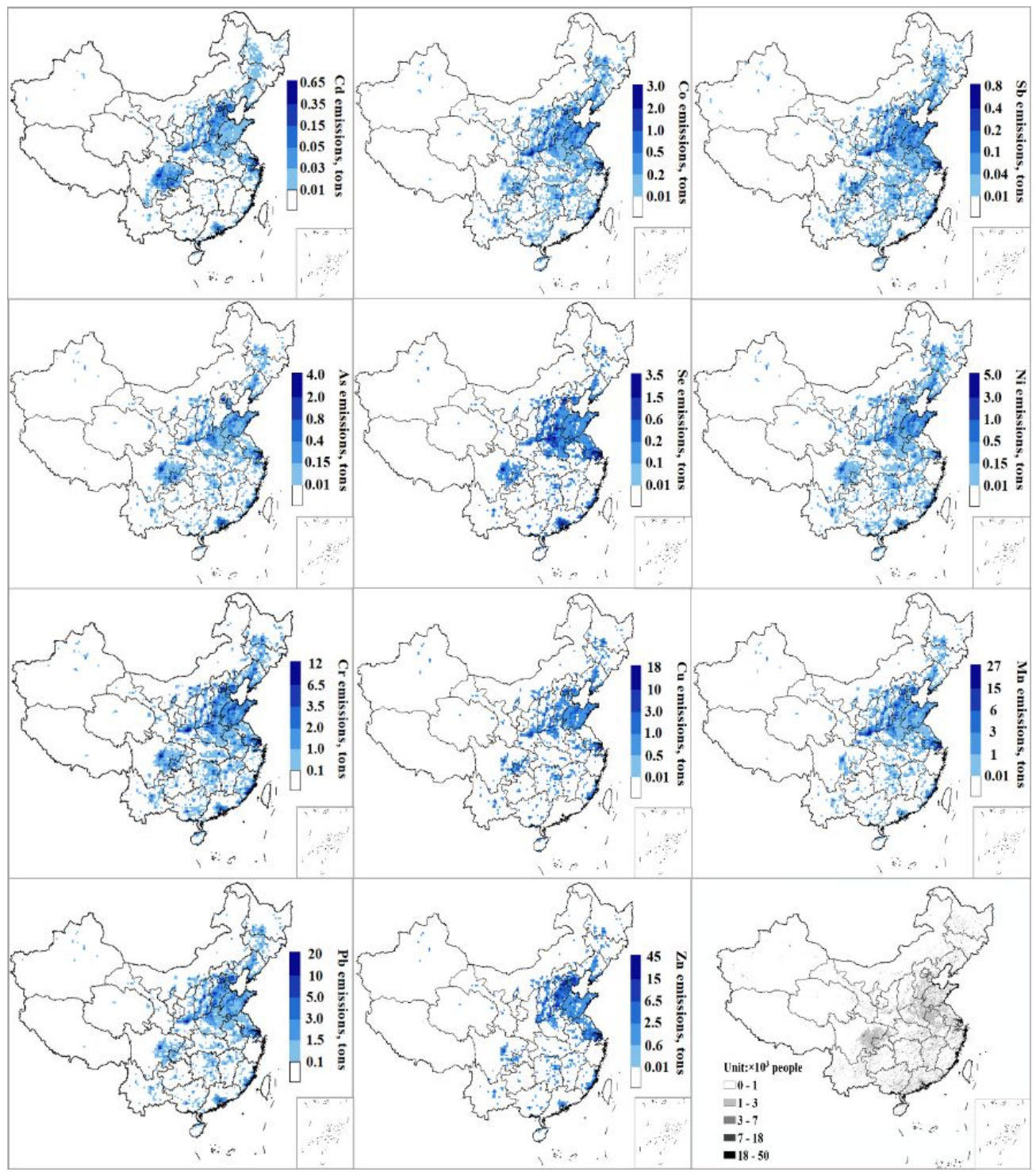

Figure S16. Spatial variations of the reductions of TEs emissions in China from 2012 to 2015 by trace element (2012 emissions minus 2015 emissions) 


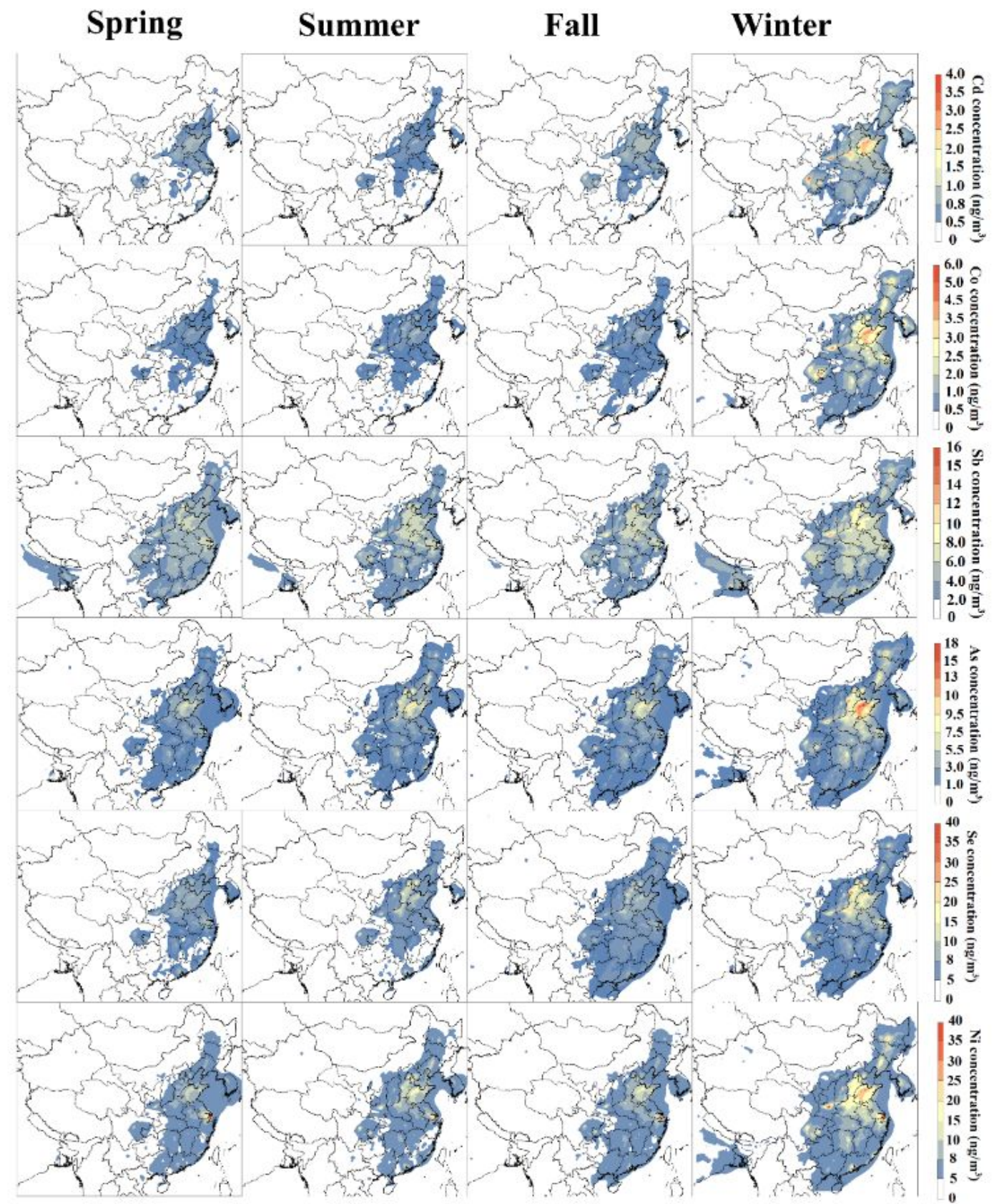

Figure S17. Spatiotemporal variations of $\mathrm{Cd}, \mathrm{Co}, \mathrm{Sb}, \mathrm{As}, \mathrm{Se}$ and $\mathrm{Ni}$ in China, 2012. Spatial distribution of averaged seasonal concentrations of Cd, Co, Sb, As, Se and Ni in China, 2012 


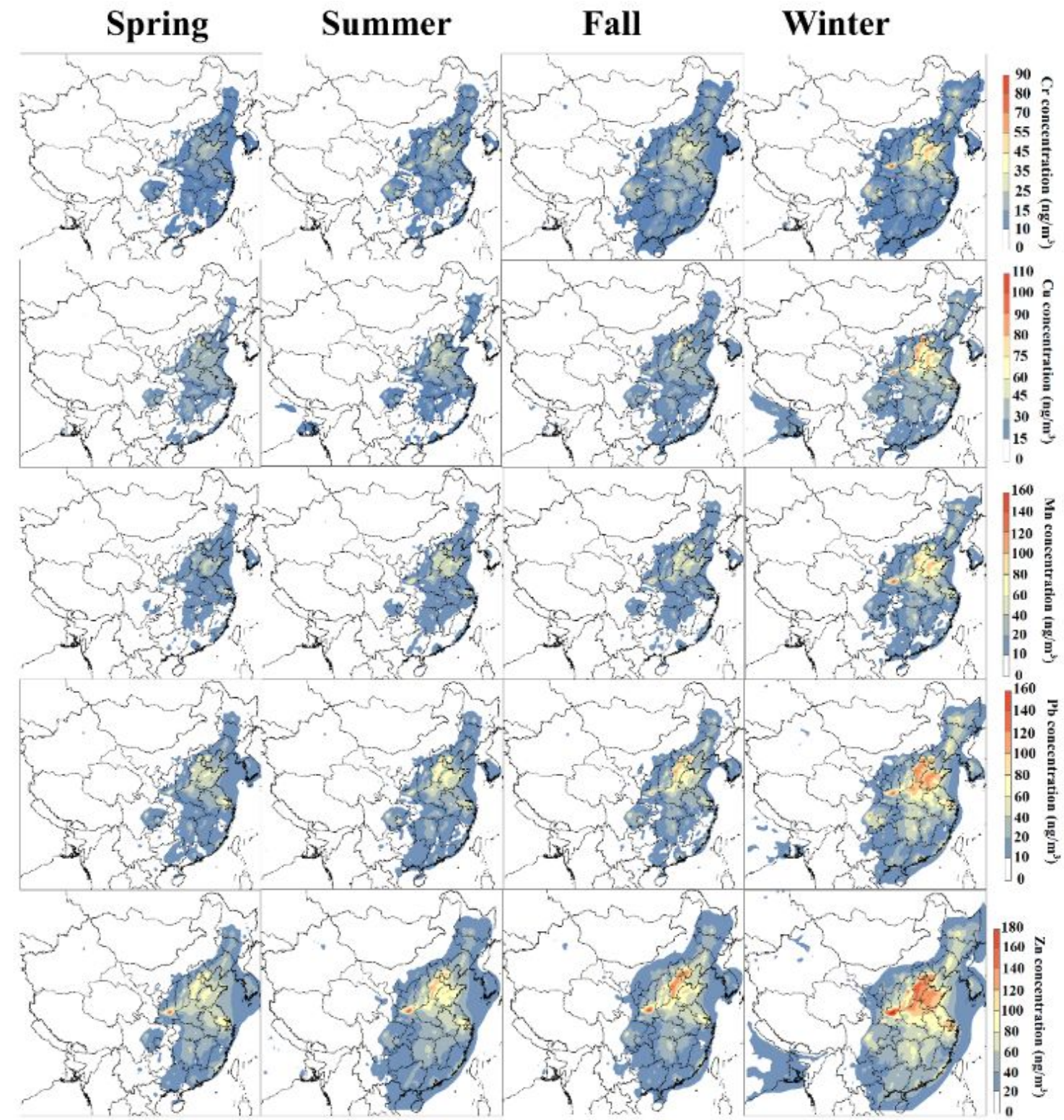

Figure S18. Spatiotemporal variations of $\mathrm{Cr}, \mathrm{Cu}, \mathrm{Mn}, \mathrm{Pb}$ and $\mathrm{Zn}$ in China, 2012. Spatial distribution of averaged seasonal concentrations of $\mathrm{Cr}, \mathrm{Cu}, \mathrm{Mn}, \mathrm{Pb}$ and $\mathrm{Zn}$ in China, 2012 


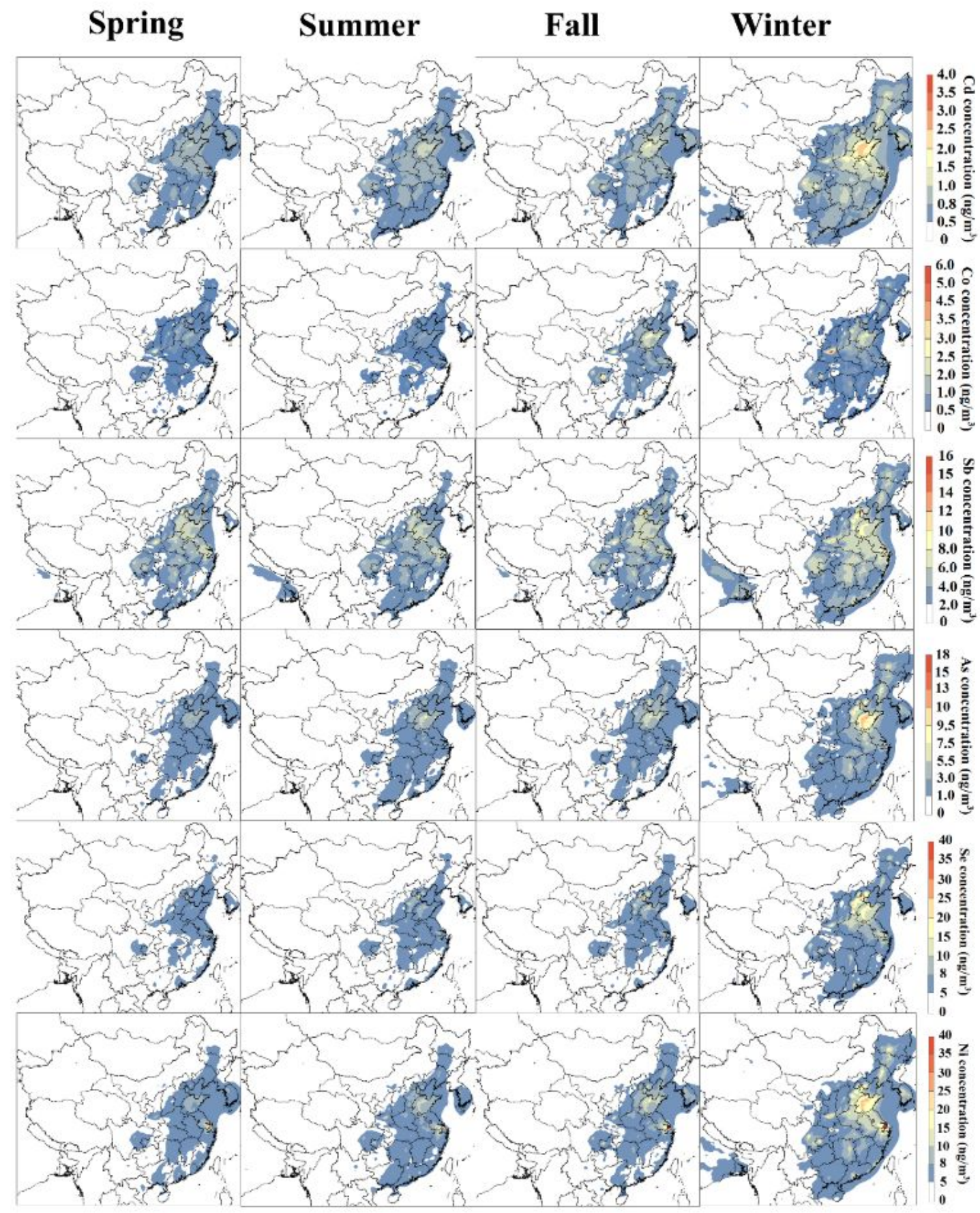

Figure S19. Spatiotemporal variations of $\mathrm{Cd}, \mathrm{Co}, \mathrm{Sb}, \mathrm{As}$, Se and Ni in China, 2015. Spatial distribution of averaged seasonal concentrations of $\mathrm{Cd}, \mathrm{Co}, \mathrm{Sb}, \mathrm{As}$, Se and Ni in China, 2015 


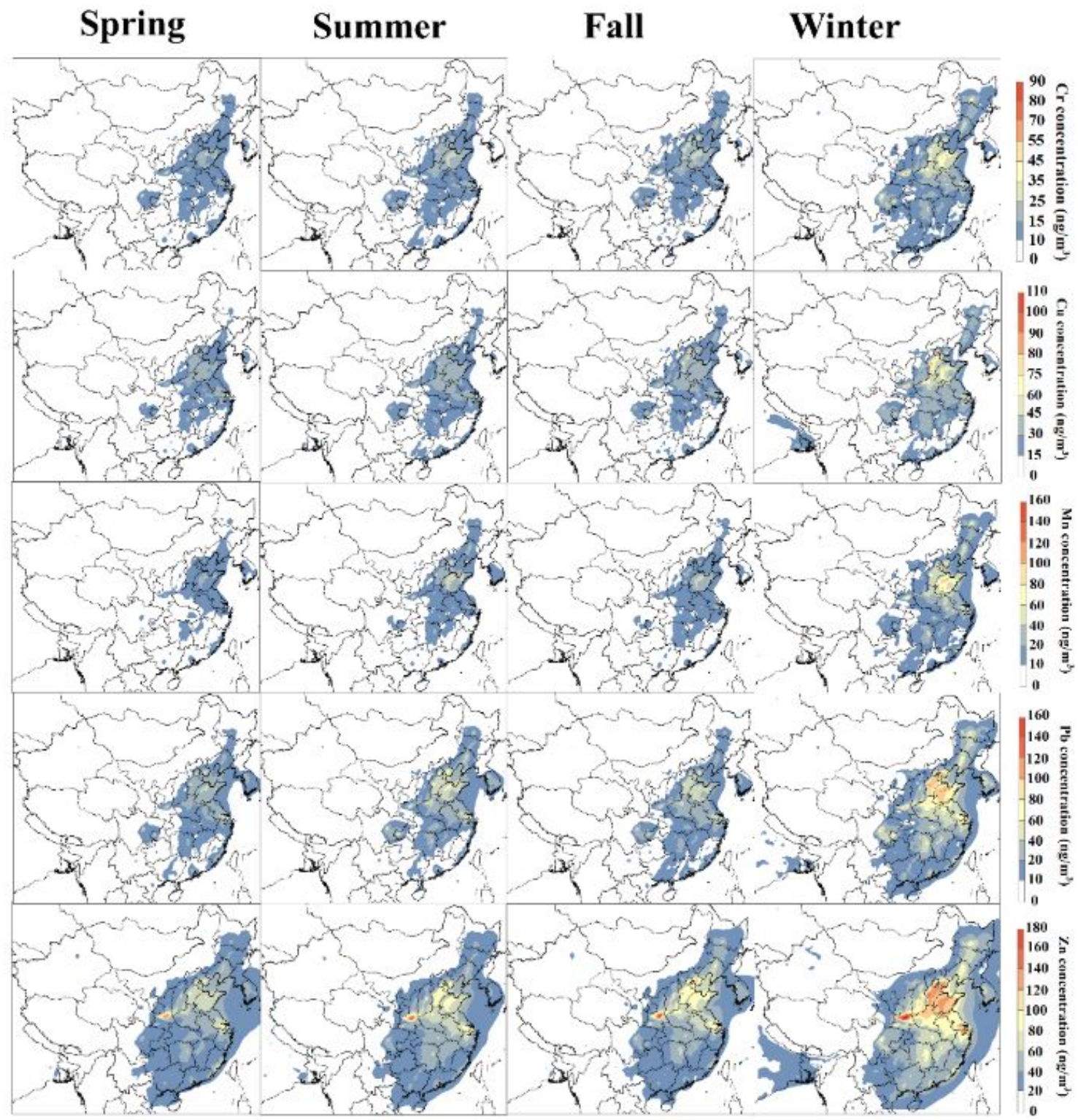

Figure S20. Spatiotemporal variations of $\mathrm{Cr}, \mathrm{Cu}, \mathrm{Mn}, \mathrm{Pb}$ and $\mathrm{Zn}$ in China, 2015. Spatial distribution of averaged seasonal concentrations of $\mathrm{Cr}, \mathrm{Cu}, \mathrm{Mn}, \mathrm{Pb}$ and $\mathrm{Zn}$ in China, 2015 

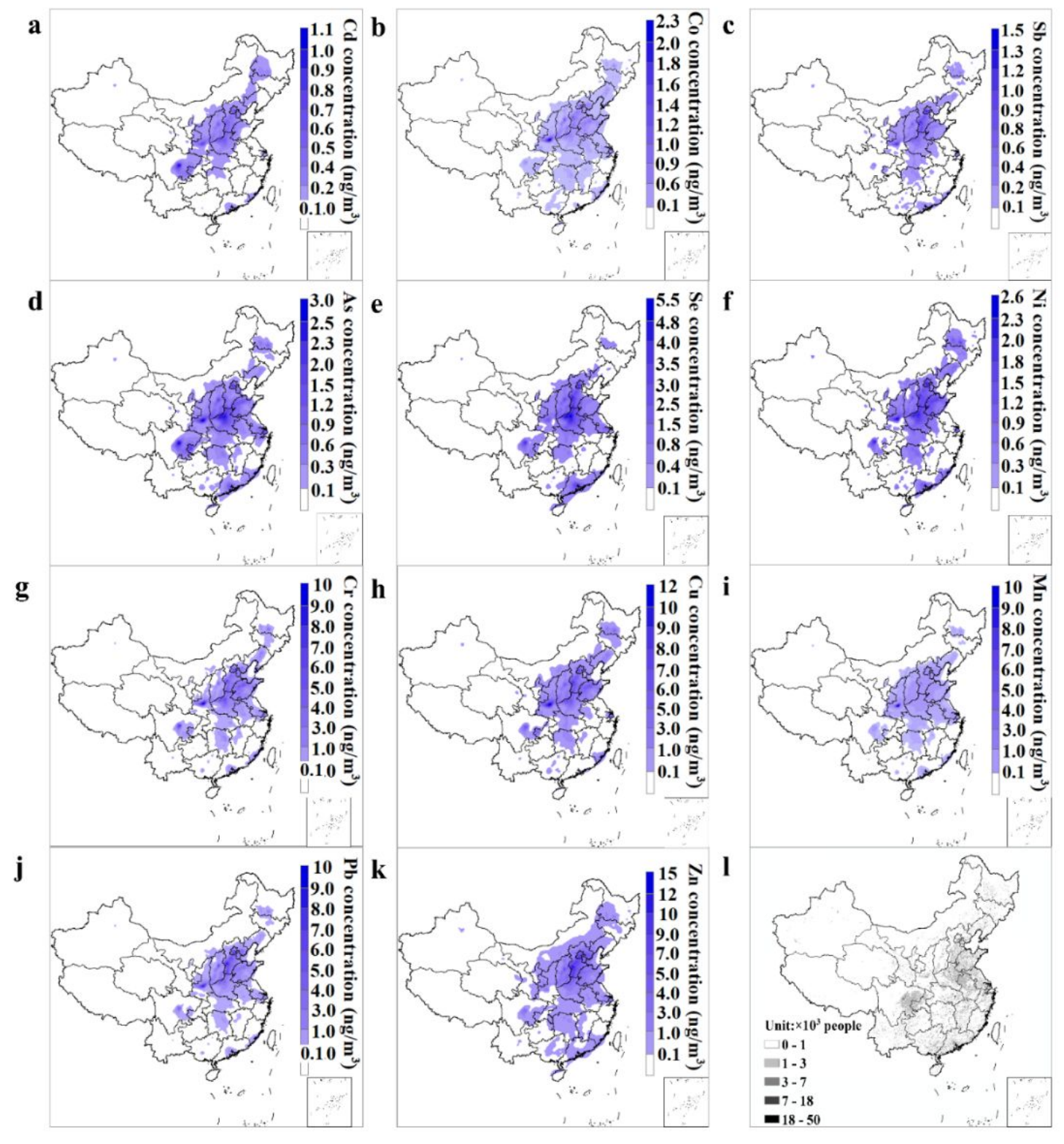

g
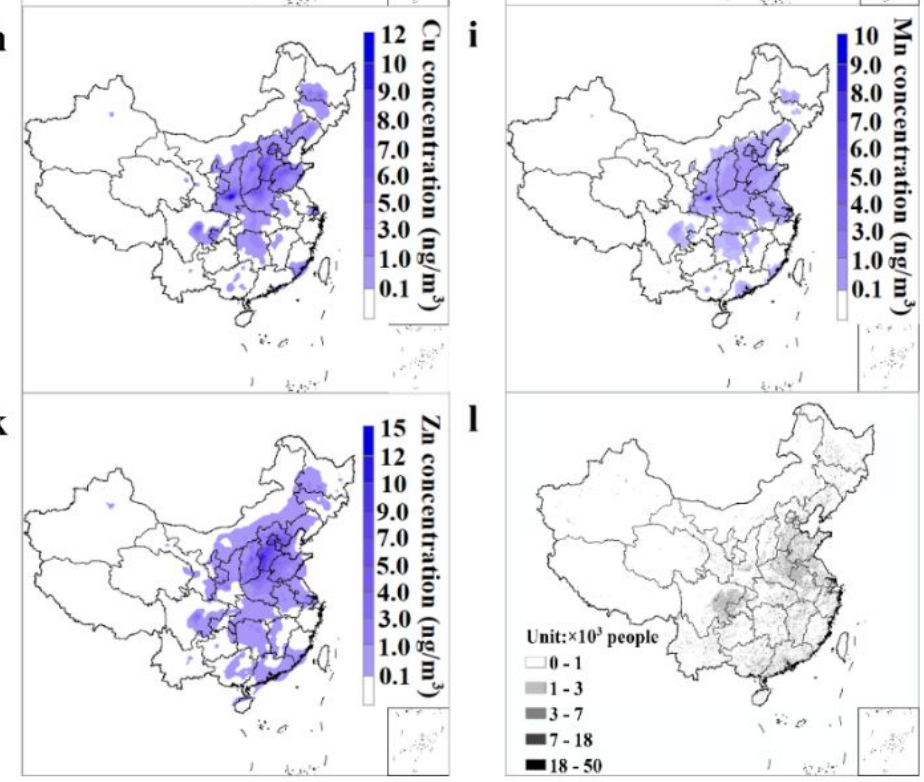

Figure S21. Spatial variations of TEs concentrations in China from 2012 to 2015. The spatial distribution of concentration reductions of each trace element from 2012 to 2015 (2012 concentrations minus 2015 concentrations) 


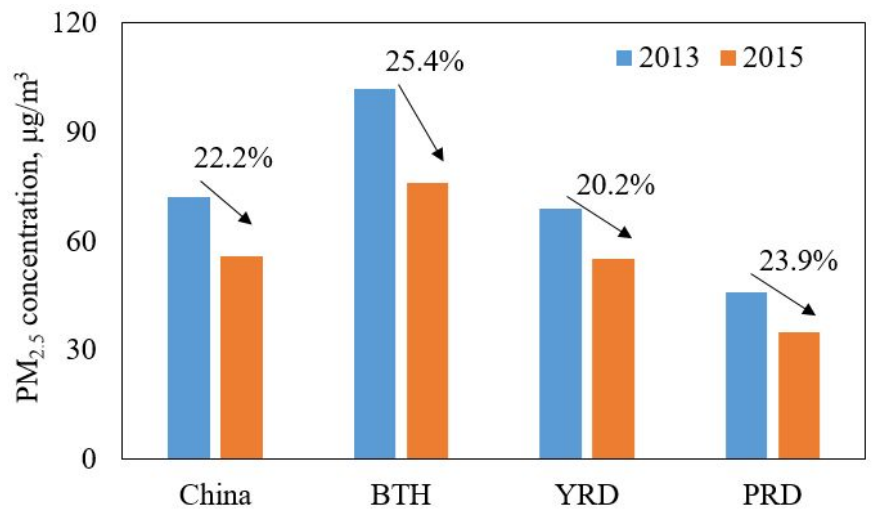

Figure S22. Annual surface observed $\mathrm{PM}_{2.5}$ concentrations of China, BTH, YRD, and PRD region from 2013 to 2015. 


\section{ILCR value for adults in 2012 ILCR value for children in 2012}

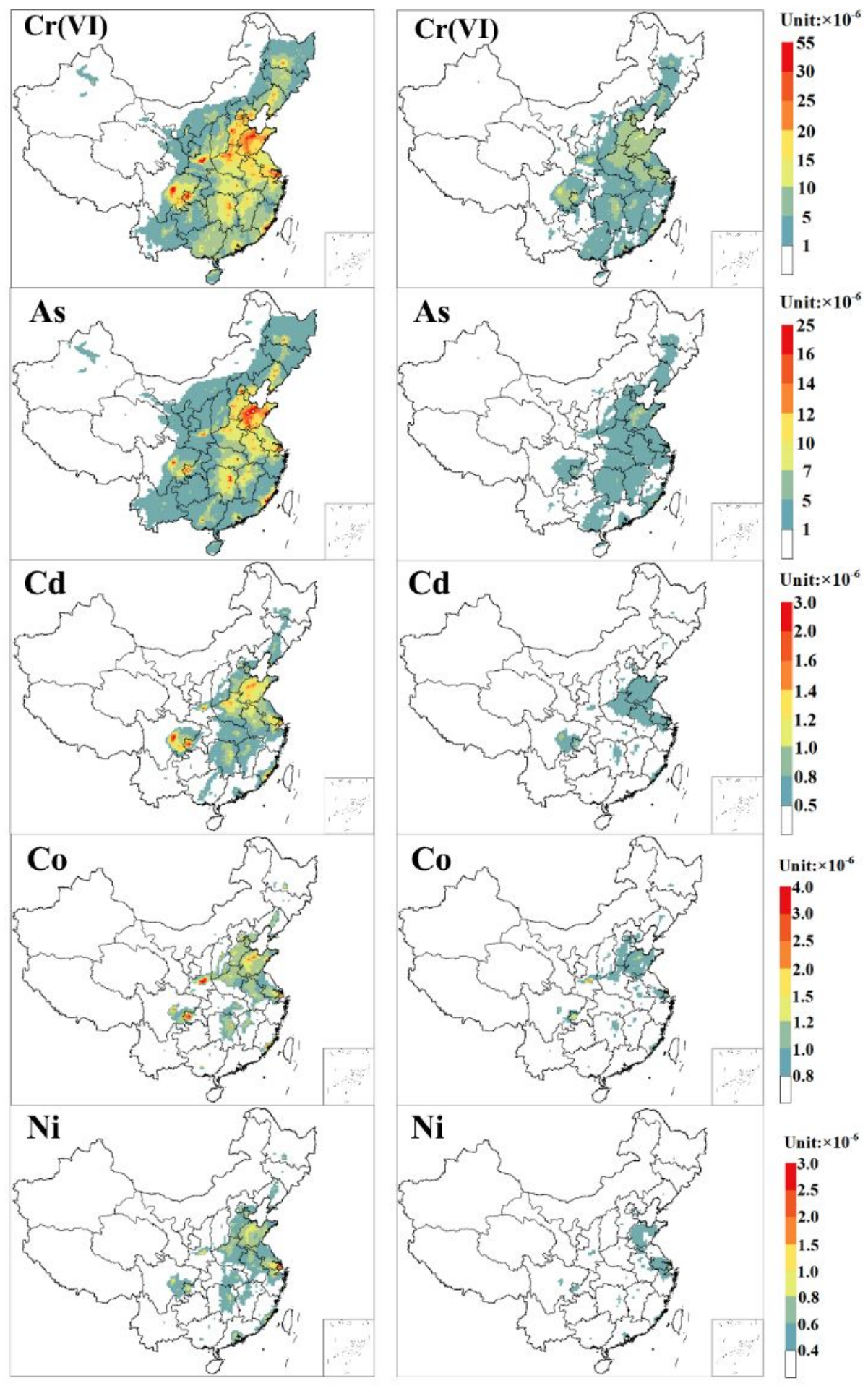

Figure S23. Spatial distribution of ILCR value in 2012 for adults and children. 


\section{ILCR value for adults in 2015}
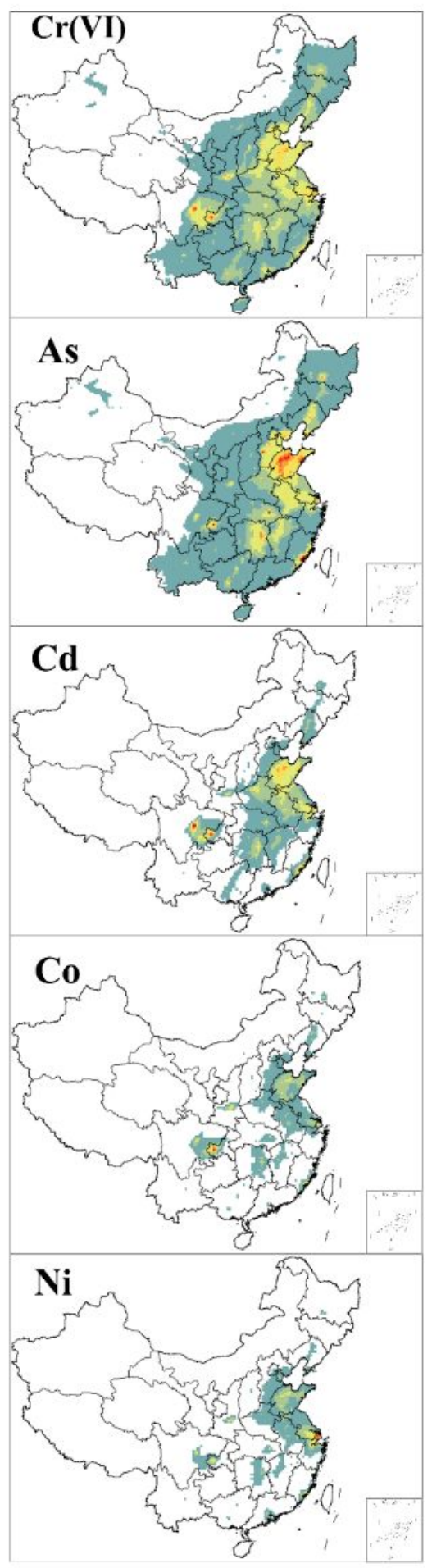

\section{ILCR value for Children in 2015}
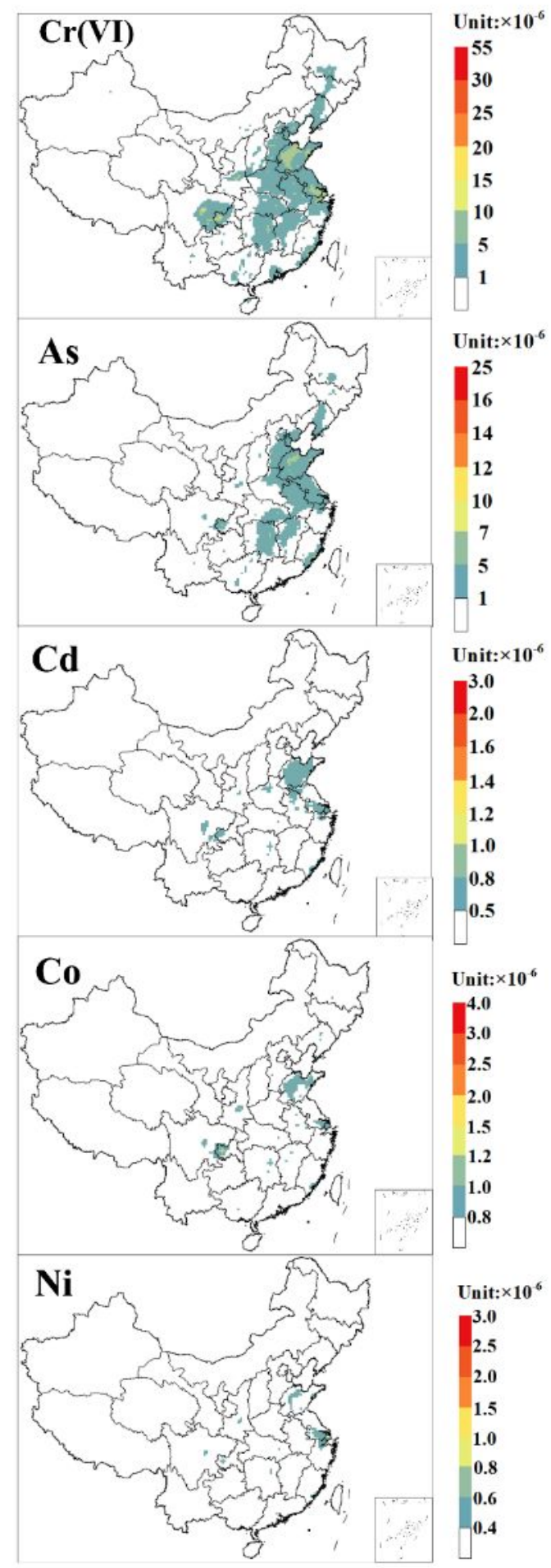

Unit: $\times 10^{6}$

Figure S24. Spatial distribution of ILCR value in 2015 for adults and children 
HI value for adults in 2012

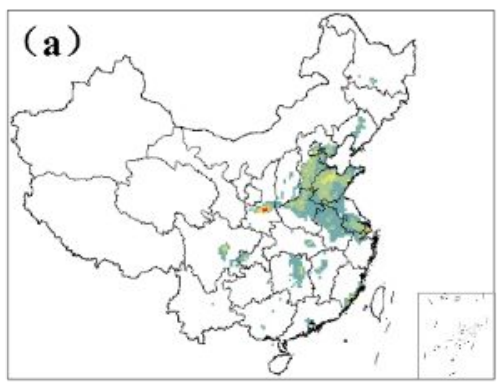

HI value for adults in 2015

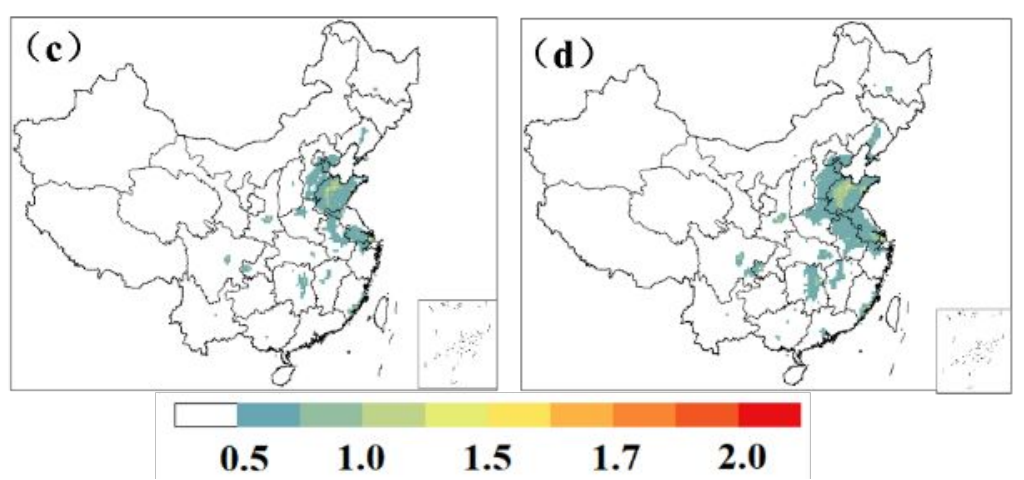

HI value for children in 2012

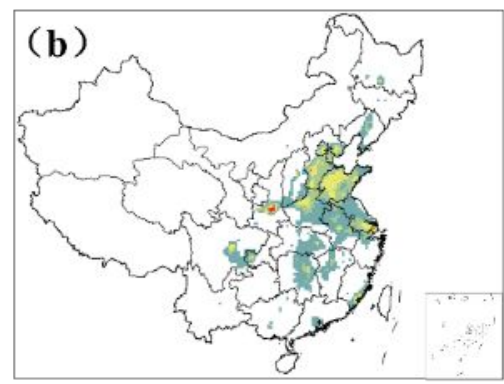

HI value for children in 2015

Figure S25. Spatial distribution of HI value in 2012 and 2015 


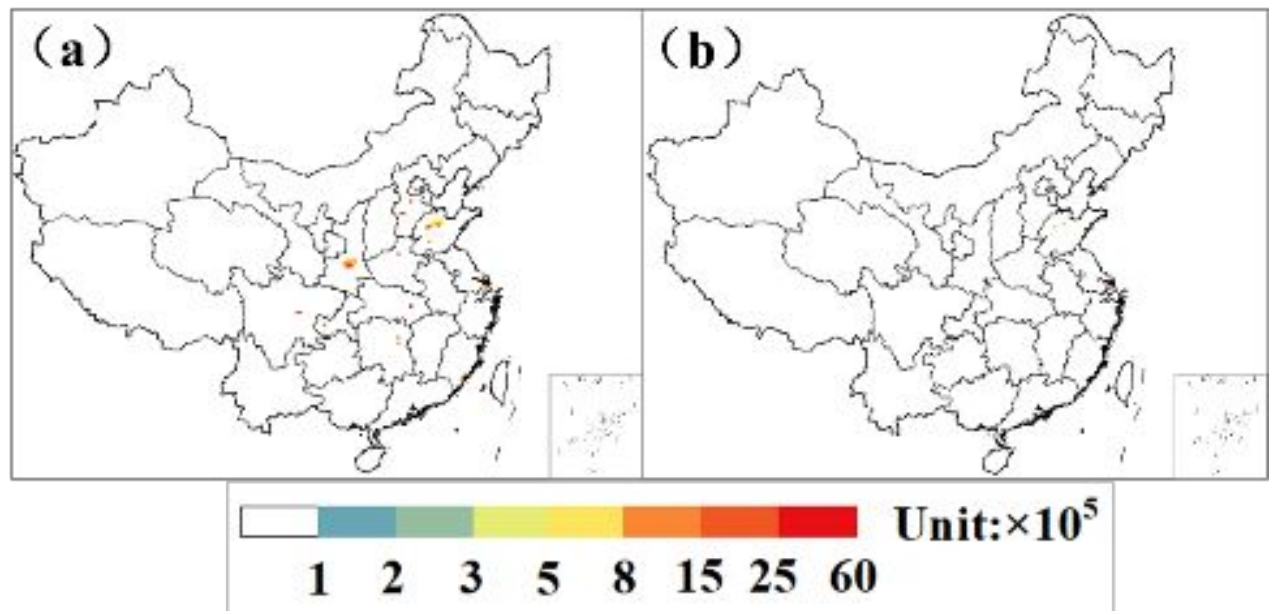

Figure S26. Spatial distribution of people lived in the environment with HI exceeding 1 by TEs inhalation in 2012 (a) and 2015 (b) 


\section{SI References}

(1) Tian, H.; Zhu, C.; Gao, J.; Cheng, K.; Hao, J.; Wang, K.; Hua, S.; Wang, Y.; Zhou, J. Quantitative assessment of atmospheric emissions of toxic heavy metals from anthropogenic sources in China: Historical trend, spatial variation distribution, uncertainties and control policies. Atmos. Chem. Phys. 2015, 15, 12107-12166.

(2) Chen, M.; Gong, Y.; Li, Y.; Lu, D.; Zhang, H. Population distribution and urbanization on both sides of the Hu Huanyong Line: Answering the Premier's question. J. Geophys. Res. 2016, 26, 15931610.

(3) Hu, J.; Chen, J.; Ying, Q.; Zhang, H. One-year simulation of ozone and particulate matter in china using WRF/CMAQ modeling system. Atmos. Chem. Phys. 2016, 16, 10333-10350.

(4) Fu, X.; Wang, T.; Gao, J.; Wang, P.; Liu, Y.; Wang, S.; Zhao, B.; Xue, L. Persistent heavy winter nitrate pollution by increased photochemical oxidants in Northern China. Environ. Sci. Technol. 2020, 54, 3881-3889.

(5) Ying, Q.; Zhang, J.; Zhang, H.; Hu, J.; Kleeman, M. J. Atmospheric age distribution of primary and secondary inorganic aerosols in a polluted atmosphere. Environ. Sci. Technol. 2021, 55, 5668-5676.

(6) Gao J.; Wang, K.; Wang Y.; Liu, S.; Zhu, C.; Hao, J.; Liu, H.; Hua, S.; Tian, H. Temporalspatial characteristics and source apportionment of $\mathrm{PM}_{2.5}$ as well as its associated chemical species in the Beijing-Tianjin-Hebei region of China. Environ. Pollut. 2018, 233, 714-724.

(7) Zhang, Q.; Zheng, Y.; Tong, D.; Shao, M.; Wang, S.; Zhang, Y.; Xu, X.; Wang, J.; He, H.; Liu, W.; Ding, Y.; Lei, Y.; Li, J.; Wang, Z.; Zhang, X.; Wang, Y.; Cheng, J.; Liu, Y.; Shi, Q.; Yan, L.; Geng, G.; Hong, C.; Li, M.; Liu, F.; Zheng, B.; Cao, J.; Ding, A.; Gao, J.; Fu, Q.; Huo, J.; Liu, B.; Liu, Z.; Yang, F.; He, K.; Hao, J. Drivers of improved $\mathrm{PM}_{2.5}$ air quality in China from 2013 to 2017. Proc. Natl. Acad. Sci. U.S.A. 2020, 116, 24463-24469.

(8) Duan, J.; Tan, J. Atmospheric heavy metals and Arsenic in China: Situation, sources and control policies. Atmos. Environ. 2013, 74, 93-101.

(9) Yang, Q.; Zhang, K.; Chai, F.; Li, J.; Zhou, G.; Zhen, Z. Study on the pollution characteristics of atmospheric particles and heavy matals in autumn in Chang-Zhu-Tan metropolitan area. Res. Environ. Sci. 2013, 26, 590-597.

(10) Zou, T.; Kang, W.; Zhang J.; Wang, M.; Fang, X.; Xue, S.; Zhong, B. Concentrations and distribution characteristics of atmospheric heavy metals in urban areas of China. Res. Environ. Sci. 2015, 28, 1053-1061.

(11) Li, H.; Wang, J.; Wang, Q.; Qian, X.; Qian, Y.; Yang, M.; Li, F.; Lu, H.; Wang, C. Chemical fractionation of arsenic and heavy metals in fine particle matter and its implications for risk assessment: A case study in Nanjing, China. Atmos. Environ. 2015, 103, 339-346. 
(12) Wang, J.; Pan, Y.; Tian, S.; Chen, X.; Wang, L.; Wang, Y. Size distributions and health risks of particulate trace elements in rural areas in northeastern China. Atmos. Res. 2016, 168, 191-204.

(13) Chen, X. Pollution Characteristics of heavy metals in atmospheric particulate matter in typical area. Shanxi University, 2016.

(14) Qiao, B. Analysis on the pollution characteristics and sources of metal elements in $\mathrm{PM}_{2.5}$ in Beijing-Tianjin-Hebei region. Beijing University of Chemical Technology, 2017.

(15) Tian, R. Pollution characteristics and source apportionment of $\mathrm{PM}_{2.5}$ in Hengyang city. University of South China, 2017.

(16) He, Z.; Wang, X.; Zhang, H.; Wang, X.; Li, B.; Guo, S. Source analysis of $\mathrm{PM}_{2.5}$ particles in Long gang district, Shenzhen city. J. Appl. Prev. Med. 2017, 23, 363-366.

(17) Chen, R.; Li, Y.; Yang, H. Monitoring of atmospheric fine particulate matter composition in Lanzhou city in 2015. J. Environ. Health 2017, 34, 231-233.

(18) Zhang, L.; Fan, F.; Jiang, W.; Cao, T.; Zhang, W.; Qi, J. Concentrations and health risk assessment of heavy metals in atmospheric $\mathrm{PM}_{2.5}$ in the Pearl River Delta Region. Acta Sci. Circumst. 2017, 37, 370-380.

(19) Fu, X.; Peng, X.; Wang, Y.; Zhou, C.; Liang, X.; Tan, Y.; Li, Z.; Dai, W. Characteristic of elements in $\mathrm{PM}_{2.5}$ and health risk assessment of heavy metals in Guangzhou. J. Environ. Health 2018, $35,154-158$.

(20) Ma, J. Element analysis and source analysis of fine particulate matter in Shanghai atmosphere. Shanghai Normal University, 2018.

(21) Yang, X. Analysis of elemental pollution characteristics and health risk assessment of $\mathrm{PM}_{2.5}$ in different cities. Shanxi University, 2019.

(22) Liu, C. Characteristic Analysis and risk assessment of typical heavy metal pollutants in urban dust in Shenyang city. Shenyang University, 2019.

(23) $\mathrm{Gu}, \mathrm{J}$. A review on heavy metals in atmospheric suspended particles of china cities and its implication for future references. Earth Environ. 2019, 47, 385-395.

(24) Liu, S.; Zhu, C.; Tian, H.; Wang, Y.; Zhang, K.; Wu, B.; Liu, X.; Hao, Y.; Liu, W.; Bai, X.; Lin, S.; Wu, Y.; Shao, P.; Liu, H. Spatiotemporal variations of ambient concentrations of trace elements in a highly polluted region of China. J. Geophys. Res-Atmos. 2019, 124, 4186-4202.

(25) Zhao, Y.; Wang, S.; Duan, L.; Lei, Y.; Cao, P.; Hao, J. Primary air pollutant emissions of coalfired power plants in China: Current status and future prediction. Atmos. Environ. 2008, 42, 8442-8452.

(26) Tian, H.; Lu, L.; Cheng, K.; Hao, J.; Zhao, D.; Wang, Y.; Jia, W.; Qiu, P. Anthropogenic atmospheric nickel emissions and its distribution characteristics in China. Sci. Total Environ. 2012, 417, $148-157$.

(27) Zhao, Y.; Nielsen, C. P.; Lei, Y.; McElroy, M. B.; Hao, J. Quantifying the uncertainties of a bottom-up emission inventory of anthropogenic atmospheric pollutants in China. Atmos. Chem. Phys. 
2011, 11, 2295-2308.

(28) U.S. Environmental Protection Agency (EPA), Risk Assessment Guidance for Superfund. In: Part A: Human Health Evaluation Manual; Part E, Supplemental Guidance for Dermal Risk Assessment; Part F, Supplemental Guidance for Inhalation Risk Assessment, 2011.

(29) Cao, S.; Duan, X.; Zhao, X.; Ma, J.; Dong, T.; Huang, N.; Sun, C.; He, B.; Wei, F. Health risks from the exposure of children to $\mathrm{As}, \mathrm{Se}, \mathrm{Pb}$ and other heavy metals near the largest coking plant in China. Sci. Total Environ. 2014, 472, 1001-1009.

(30) Hu, X.; Zhang, Y.; Ding, Z.; Wang, T.; Lian, H.; Sun, Y.; Wu, J. Bioaccessibility and health risk of arsenic and heavy metals (cd, co, cr, cu, ni, pb, zn and mn) in tsp and $\mathrm{PM}_{2.5}$ in Nanjing, China. Atmos. Environ. 2012, 57, 146152.

(31) U.S. EPA (United States Environmental Protection Agency). Integrated Risk Information System (IRIS) [EB/OL]. https://iris.epa.gov/AtoZ/?list_type=alpha. 